\title{
A multi-wavelength census of star formation activity in the young embedded cluster around Serpens/G3-G6 ${ }^{\star}, \star \star$
}

\author{
A. A. Djupvik ${ }^{1, \star \star \star}$, Ph. André ${ }^{2,5}$, S. Bontemps ${ }^{3}$, F. Motte ${ }^{2,5}$, G. Olofsson ${ }^{4}$, M. Gålfalk ${ }^{4}$, and H.-G. Florén ${ }^{4}$ \\ 1 Nordic Optical Telescope, Apdo 474, 38700 Santa Cruz de La Palma, Spain \\ e-mail: amanda@not.iac.es \\ 2 CEA/DSM/DAPNIA, Service d'Astrophysique, C.E. Saclay, Orme des Merisiers, 91191 Gif-sur-Yvette, France \\ 3 Observatoire de Bordeaux, BP 89, 33270 Floirac, France \\ ${ }^{4}$ Stockholm Observatory, Roslagstullsbacken 21, 10691 Stockholm, Sweden \\ 5 AIM - Unité Mixte de Recherche CEA - CNRS - Université Paris VII - UMR 7158, France
}

Received 3 May 2006 / Accepted 6 July 2006

\section{ABSTRACT}

\begin{abstract}
Aims. The aim of this paper is to characterise the star formation activity in the poorly studied embedded cluster Serpens/G3-G6, located $\sim 45^{\prime}(3 \mathrm{pc}$ ) to the south of the Serpens Cloud Core, and to determine the luminosity and mass functions of its population of Young Stellar Objects (YSOs).

Methods. Multi-wavelength broadband photometry was obtained to sample the near and mid-IR spectral energy distributions to separate YSOs from field stars and classify the YSO evolutionary stage. ISOCAM mapping in the two filters $L W 2(5-8.5 \mu \mathrm{m})$ and $L W 3$ $(12-18 \mu \mathrm{m})$ of a $19^{\prime} \times 16^{\prime}$ field was combined with $J H K_{\mathrm{S}}$ data from $2 \mathrm{MASS}, K_{\mathrm{S}}$ data from Arnica/NOT, and $L^{\prime}$ data from SIRCA/NOT. Continuum emission at $1.3 \mathrm{~mm}$ (IRAM) and $3.6 \mathrm{~cm}$ (VLA) was mapped to study the cloud structure and the coldest/youngest sources. Deep narrow band imaging at the $2.12 \mu \mathrm{m} \mathrm{S}$ (1) line of $\mathrm{H}_{2}$ from NOTCam/NOT was obtained to search for signs of bipolar outflows. Results. We have strong evidence for a stellar population of 31 Class II sources, 5 flat-spectrum sources, 5 Class I sources, and two Class 0 sources. Our method does not sample the Class III sources. The cloud is composed of two main dense clumps aligned along a ridge over $\sim 0.5 \mathrm{pc}$ plus a starless core coinciding with absorption features seen in the ISOCAM maps. We find two S-shaped bipolar collimated flows embedded in the NE clump, and propose the two driving sources to be a Class 0 candidate (MMS3) and a double Class I (MMS2). For the Class II population we find a best age of $\sim 2$ Myr and compatibility with recent Initial Mass Functions (IMFs) by comparing the observed Class II luminosity function (LF), which is complete to $0.08 L_{\odot}$, to various model LFs with different star formation scenarios and input IMFs.
\end{abstract}

Key words. stars: formation - stars: pre-main sequence - stars: luminosity function, mass function - ISM: jets and outflows stars: low-mass, brown dwarfs

\section{Introduction}

The Serpens Cauda Clouds are among the darkest regions in a complex of clouds called the Aquila Rift (Dame \& Thaddeus 1985). The young embedded cluster in the Serpens Cloud Core has been well studied at most wavelengths, see Eiroa et al. (2006) for a recent review. About $45^{\prime}$ to the south there is also a region with active star formation. Cohen \& Kuhi (1979) found 4 optical T Tauri stars in a $30^{\prime \prime}$ field and named them Ser/G3-G6. Clark (1990, 1991) mapped the area surrounding Ser/G3-G6 in the ammonia 1, 1 emission line and found two $\mathrm{NH}_{3}$ cores, one on each side of the optically visible stellar group: Ser/G3-6NE and Ser/G3-G6SW. Ziener \& Eislöffel (1999) surveyed 3.14 square degrees in the optical lines of [S II] and found the Herbig-Haro object HH476 close to the Ser/G3-G6SW core. The energy source of HH476 was identified by Wu et al. (2002) to be IRAS 18265+0028. At this position Persi et al. (1994) found

* Based on observations with ISO, an ESA project with instruments funded by ESA Member States (especially the PI countries: France, Germany, The Netherlands, and the UK) and with the participation of ISAS and NASA.

$\star \star$ Tables 1-3 are only available in electronic form at the CDS via anonymous ftp to cdsarc.u-strasbg.fr (130.79.128.5) or via http://cdsweb.u-strasbg.fr/cgi-bin/qcat?J/A+A/458/789 $\star \star \star$ Changed family name from Kaas to Djupvik. a $\mathrm{H}_{2} \mathrm{O}$ maser. Recently, Harvey et al. (2006) found 24 YSO candidates in a $12^{\prime} \times 12^{\prime}$ area in this region (which they refer to as Cluster B) from a mid-IR survey with Spitzer.

When preparing the ISOCAM star formation survey ${ }^{1}$, this region was defined based on the ammonia maps of Clark (1990) and named Serpens NH3. The field is relatively opaque at optical wavelengths and has a peak brightness at $60 \mu \mathrm{m}$ (Zhang et al. 1988).

We will use the distance of $225 \pm 55 \mathrm{pc}$ to the Serpens Cauda Clouds according to Straižys et al. (2003), keeping in mind the caveat of the same authors that the cloud complex is possibly 80 pc deep.

Preliminary ISOCAM results from this region were presented in Kaas et al. (1999) and Kaas (1999b). In this paper we present the full results of a $17^{\prime} \times 19^{\prime}$ ISOCAM survey in LW2 $(6.7 \mu \mathrm{m})$ and LW3 $(14.3 \mu \mathrm{m})$, cross-correlated with the 2MASS point source catalogue. In addition we present deep follow-up $\mathrm{H}_{2}$ line $(2.122 \mu \mathrm{m}), K_{\mathrm{S}}$ band $(2.15 \mu \mathrm{m})$, and $L^{\prime}$ band $(3.8 \mu \mathrm{m})$ imaging from the Nordic Optical Telescope, as well as IRAM $1.3 \mathrm{~mm}$ continuum and VLA $3.6 \mathrm{~cm}$ mapping of the most active part of the region.

\footnotetext{
${ }^{1}$ One of the guaranteed time programs with the camera aboard the Infrared Space Observatory (ISO).
} 
We describe the various observation sets with reductions and results in Sect. 2, the use of IR excesses to extract YSO candidates in Sect. 3, the classification of the YSO population in Sect. 4, the luminosity and mass function in Sect. 5, and the cloud structure and the outflow sources in Sect. 6.

\section{Observations and reductions}

\subsection{ISOCAM}

This paper is based on observations obtained with ISOCAM, the camera aboard the Infrared Space Observatory (ISO; see Kessler et al. 1996; Cesarsky et al. 1996), and is part of the LNORDH.SURVEY_1 star formation survey conducted in the two broadband filters LW2 $(5-8.5 \mu \mathrm{m})$ and LW3 $(12-18 \mu \mathrm{m})$, see, e.g., Kaas \& Bontemps (2000) for a review. In the region called Serp-NH3 about 0.09 square degrees were surveyed in LW2 and LW3 in 1996. The pixel field of view (PFOV) was 6" and the unit integration time $0.28 \mathrm{~s}$. The region was mapped by rastering along right ascension with about half a frame $\left(90^{\prime \prime}\right)$ overlap in $\alpha$ and $24^{\prime \prime}$ overlap in $\delta$, producing a 12 by 6 mosaic. Each position in the sky was observed for about $15 \mathrm{~s}$. Image reductions were performed using $\mathrm{CIA}^{2}$ and a set of our own programs for point source detection and photometry. We refer to Kaas et al. (2004) for a detailed description of the data reduction. The fluxes in ADU/s in LW2 and LW3 are converted to $\mathrm{mJy}$ through the relations 2.32 and $1.96 \mathrm{ADU} / \mathrm{gain} / \mathrm{s} / \mathrm{mJy}$ for LW2 and LW3, respectively (Blommaert et al. 2000). These are strictly valid only for $F_{v} \propto v^{-1}$ sources and a small colour correction has been applied to the "blue" sources, which have significantly different spectra $\left(F_{v} \propto v^{-3}\right)$. The reference wavelengths are defined at 6.7 and $14.3 \mu \mathrm{m}$ for LW2 and LW3, respectively. Conversion from flux density to magnitude is defined as $m_{6.7}=-2.5 \log \left(F_{v}(6.7 \mu \mathrm{m}) / 82.8\right)$ and $m_{14.3 \mu \mathrm{m}}=$ $-2.5 \log \left(F_{v}(14.3 \mu \mathrm{m}) / 18.9\right)$, where $F_{v}$ is given in Jy. The $\sim 5 \%$ responsivity decrease throughout orbit has not been corrected for. The ISOCAM astrometry was based on the ISO pointing only. We manually cross-correlated bright sources with 2MASS positions and found a bulk offset of 7" in Dec and 1.4" in RA. Correcting for this, the mean deviation between ISOCAM and 2MASS positions (for these bright sources) became $2.5 \pm 1.4^{\prime \prime}$.

ISOCAM point source extraction and photometry gave a total of 186 detections (see Table 1) of which 160 have reliable ${ }^{3}$ fluxes in the $6.7 \mu \mathrm{m}$ band (LW2) and 74 in the $14.3 \mu \mathrm{m}$ band (LW3). For the $14.3 \mu \mathrm{m}$ band we estimate a completeness limit of $8 \mathrm{mJy}$ and a limiting sensitivity of $3 \mathrm{mJy}$, while for the $6.7 \mu \mathrm{m}$ band the survey is complete to $6 \mathrm{mJy}$ and reaches $1 \mathrm{mJy}$. At these levels the sample is not contaminated by galaxies. See the discussion in Kaas et al. (2004) for details.

Table 1, which is available in electronic form at the CDS, lists all the 186 ISOCAM detections with fluxes and uncertainties for each band (see Sect. 3 for a description of the columns). Flux measurements have been obtained in both bands for a total of 57 sources. These belong to either of two colour populations, one group of 36 "blue" sources that have colour indices like normal photospheres, and another group of 21 "red" sources that have excess emission at $14.3 \mu \mathrm{m}$ (see Fig. 4 and Sect. 3 in general for details). Figure 1 is a sky map of the ISOCAM sources.

\footnotetext{
2 CAM Interactive Analysis, a joint development by the ESA Astrophysics Division and the ISOCAM Consortium led by the ISOCAM PI, C. Cesarsky, Direction des Sciences de la Matiere, C.E.A., France.

3 Detections without reliable flux measurements are generally sources observed on the edge of the detector.
}

The locations of the IR-excess sources, that are also labelled, are shown together with those of the "blue" (no IR-excess) sources, as well as sources with either LW2 or LW3 fluxes only. The subregions marked in this map show the location and extent of various follow-up observations: IRAM $1.3 \mathrm{~mm}$ map (large box), Arnica deep Ks imaging (medium box), and NOTCam $\mathrm{H}_{2}$ line imaging (small box). The SIRCA pointed observations are not indicated in the figure.

\section{2. $2 M A S S$}

In the region mapped by ISOCAM a total of 1803 sources was found in the 2MASS All-Sky Release Point Source Catalog (Cutri et al. 2003). The $J(1.24 \mu \mathrm{m}), H(1.66 \mu \mathrm{m})$, and $K_{\mathrm{s}}$ $(2.16 \mu \mathrm{m})$ photometry of all these has been used in this paper.

We cross-correlated the 186 ISOCAM sources with the 2MASS PSC using a search radius of $7.5^{\prime \prime}$ (i.e., 3 times the mean positional deviation between ISOCAM and 2MASS for bright sources after the global recentering, see Sect. 2.1). Only 19 ISOCAM detections had no 2MASS counterpart, and of these, three are very red and faint in the near-IR, while the remaining had been detected in only one of the two ISOCAM bands. In 29 cases we found multiple 2MASS objects within the search radius. Disregarding these, the mean positional deviation of all cross-correlations is $3.1 \pm 1.5^{\prime \prime}$ and the maximum is $6.5^{\prime \prime}$, and this is a measure of the accuracy of the ISOCAM positions. None of the 29 ISOCAM sources that are associated with multiple 2MASS sources within a $7.5^{\prime \prime}$ radius are YSO candidates with excess emission at $14.3 \mu \mathrm{m}$, although ISO-132 might be a Class II to Class III transition object (cf. Sect. 4). In the 28 cases of two 2MASS sources within the search radius, we have discarded one of them as "probably not an ISOCAM counterpart", based on separation, color, and quality flags. In one case of a quadruple (ISO-NH3-118), we have discarded 3 objects.

\section{3. $K_{s}$ band imaging with Arnica at the NOT}

In August 1996, about 4 months after the ISOCAM observations, the Arcetri Near-Infrared Camera (ARNICA) was used at the Nordic Optical Telescope (NOT), La Palma, to obtain deep $K_{\mathrm{s}}$ band $(2.15 \mu \mathrm{m})$ imaging in the most active $8^{\prime} \times 8^{\prime}$ subregion of the ISOCAM field. A 4 by 4 raster mode $(256 \times 256$ array and $0.54^{\prime \prime} /$ pix) was scanned repeatedly (dithering each scan), staring at the same position only $1 \mathrm{~min}$ at a time with a unit integration time of $1 \mathrm{~s}$, until a total on-source integration time of 5 min was obtained for the whole map. In this way the program images themselves were used to subtract the thermal sky level. Flat fields were calibrated on the decreasing/increasing twilight sky, removing thermal and stray light contributions by taking difference images. Standard stars taken from the UKIRT Faint standard list (Casali \& Hawarden 1992) and from the ARNICA list (Hunt et al. 1998) were observed throughout the night. Observation and reduction methods are the same as those described in Kaas (1999a).

Figure 2 shows the deep $K_{\mathrm{S}}$ band imaging with Arnica of an $8 \times 8$ arcmin sub-region (i.e., $18 \%$ ) of the ISOCAM area, and contours of the LW2 band $(6.7 \mu \mathrm{m})$ image are overlaid. This area covers the Ser/G3-G6NE ammonia core (Clark 1991) well, and a high extinction region is outlined by the drop in the surface density of stars in the $K_{\mathrm{S}}$ band.

Point sources were detected with daofind (threshold at $5 \sigma$ ), and aperture photometry was made using a small aperture with an aperture correction applied to compensate for flux losses in 


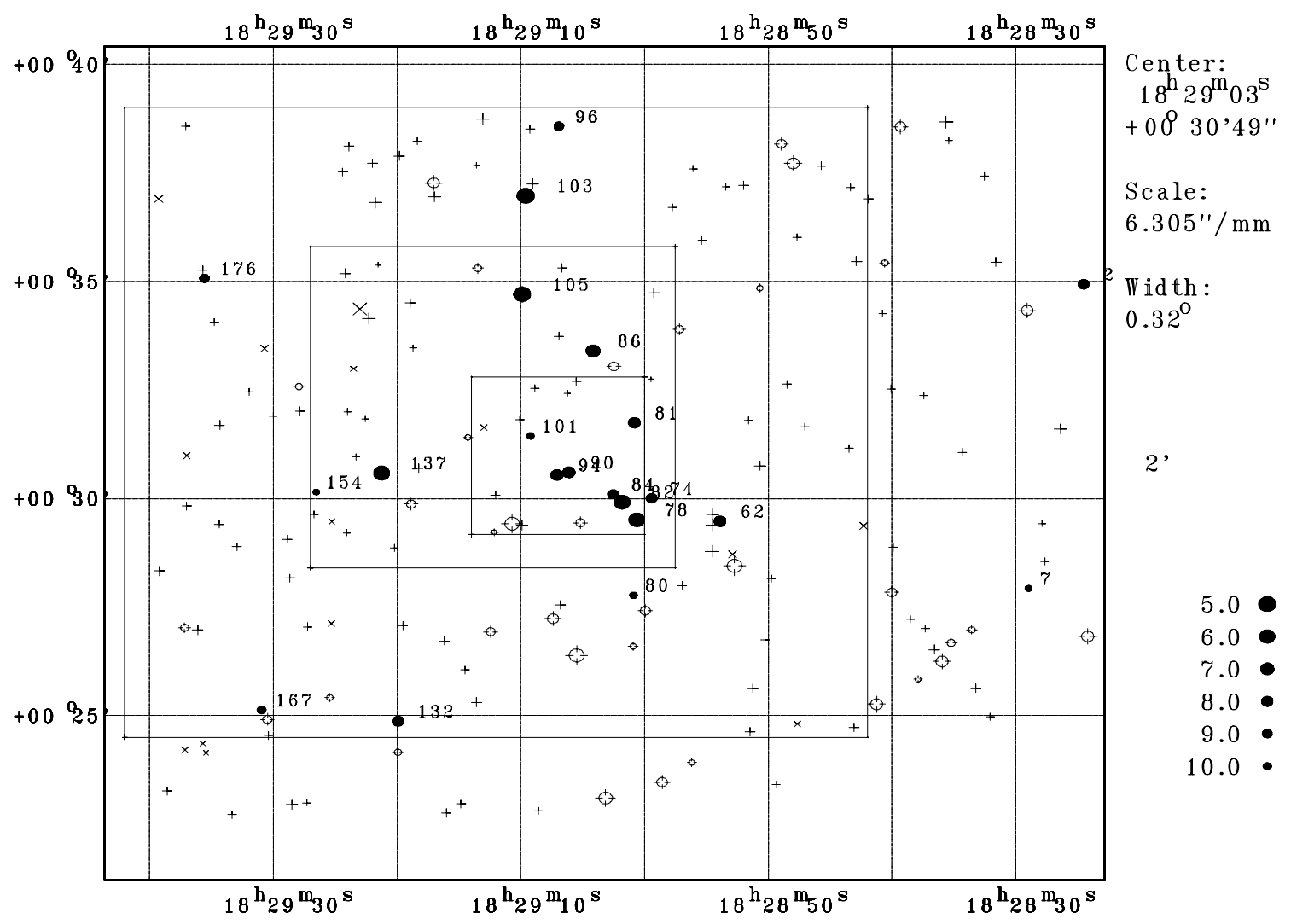

Fig. 1. Sky map of the ISOCAM sources. Symbol size inversely proportional to $L W 2$ magnitudes. IR-excess sources (filled circles), sources without IR-excess or "blue" sources (open circles), only $L W 2$ fluxes (plus sign), and only $L W 3$ fluxes (crosses). Coordinates are J2000 epoch. The three subregions indicated are the areas observed with IRAM (large box), Arnica (medium box), and NOTCam (small box). The pointed SIRCA observations are not shown. See text for details.

the wings of the stellar profile. A total of 1014 sources (out of 1148 detections) have photometry with $\sigma_{K_{\mathrm{s}}}<0.3 \mathrm{mag}$ in the $8^{\prime} \times 8^{\prime}$ Arnica map. All are listed in Table 2 (available online only). The first column gives the Arnica/NOT identification number from 1 to 1148 . The second and third columns list the $\mathbf{J} 2000$ equatorial coordinates. Columns four and five list the $K_{\mathrm{S}}$ magnitude and its error, and the last column gives possible other identifications where cross-correlation has been made with the ISOCAM map. The coordinates were found by registering the mosaic using IRAF tasks ccmap and $x y 2 r d$ with the 2MASS coordinates of 44 cross-correlated ISO and SIRCA sources as an input grid to the plate solution. The rms of the J2000 coordinates presented in Table 2 are $0.38^{\prime \prime}$ in ra and $0.31^{\prime \prime}$ in dec. This relatively large rms is probably because of the optical distortion in the camera, which was not corrected for. The sources with $\sigma_{K_{\mathrm{S}}}<0.3 \mathrm{mag}$ are in the range from $K_{\mathrm{S}}=7.5$ to $K_{\mathrm{S}}=17.8 \mathrm{mag}$, and the survey is estimated to be complete to $K_{\mathrm{S}} \approx 16 \mathrm{mag}$.

Comparing the $K_{\mathrm{S}}$ band magnitudes of Arnica/NOT (August 1996) with those of 2MASS (July 2000) for 37 ISOCAM sources in the range $7.8<K_{\mathrm{S}}<14.3$ shows overall agreement. The difference $K_{\mathrm{S}}(1996)-K_{\mathrm{S}}(2000)$ has a median value of $-0.005 \pm 0.07$ mag when disregarding six sources that have varied by more than $0.2 \mathrm{mag}$ and are therefore variable sources. These are: ISO-NH3-84 (a double Class II), ISO-NH3-86 (flat-spectrum source), ISO-NH3-94 (Class I or younger - the $K$ band emission is probably dominated by the emission in the $2.122 \mu \mathrm{m} S(1)$ line of $\mathrm{H}_{2}$ ), ISO-NH3-105 (Class II), ISO-NH3-137 (Class II), and ISO-NH3-154 (Class II). Refer to Tables 4 and 5. Because the
Arnica data was collected only 4 months after the ISOCAM observations, we use these to calculate the $\alpha_{2-7}$ and $\alpha_{2-14}$ spectral indices. Also, the Arnica dataset resolves ISO-NH3-84 into two sources and provides a $K_{\mathrm{S}}$ band magnitude for the faint source ISO-NH3-90.

\section{4. $L^{\prime}$ band imaging with SIRCA at the NOT}

The Stockholm Infrared Camera $\left(\mathrm{SIRCA}^{4}\right)$ was used at the NOT, La Palma, 15-19 July 2003. We imaged 12 SIRCA fields $\left(56^{\prime \prime} \times 70^{\prime \prime}\right)$ within the ISOCAM map in Serp-NH3. This instrument/telescope combination has a sensitivity in the $L^{\prime}$ band $(3.8 \mu \mathrm{m})$ of $0.2 \mathrm{mJy}$ per square arcsecond, i.e., a limiting magnitude of $L^{\prime}=15.2$. The detector is a $256 \times 320 \mathrm{InSb}$ array, and the spatial resolution at the NOT is $0.22^{\prime \prime} /$ pix. The images were taken with an individual integration time of $0.2 \mathrm{~s}, 5$ reads per chopper position, 5 chopper repetitions, and 20 ABBA "noddings" of the telescope, i.e., a total on-source integration time of $0.2 \times 5 \times 2 \times 5 \times 20 \times 4=800 \mathrm{~s}$. The bright UKIRT standards HD161903 and HD203856 with $L^{\prime}$ magnitudes 7.01 and 6.84 (Casali \& Hawarden 1992), respectively, were integrated $80 \mathrm{~s}$ (only two telescope noddings). The flatfield was obtained from the target frames by chopping between the sky and outside the field, i.e., the cold interior of the instrument, which effectively

\footnotetext{
4 See Olofsson et al. (1999) and van der Bliek et al. (2000), as well as URL http://www.astro.su.se/groups/ infrared/instrumentation.html for more information on SIRCA.
} 


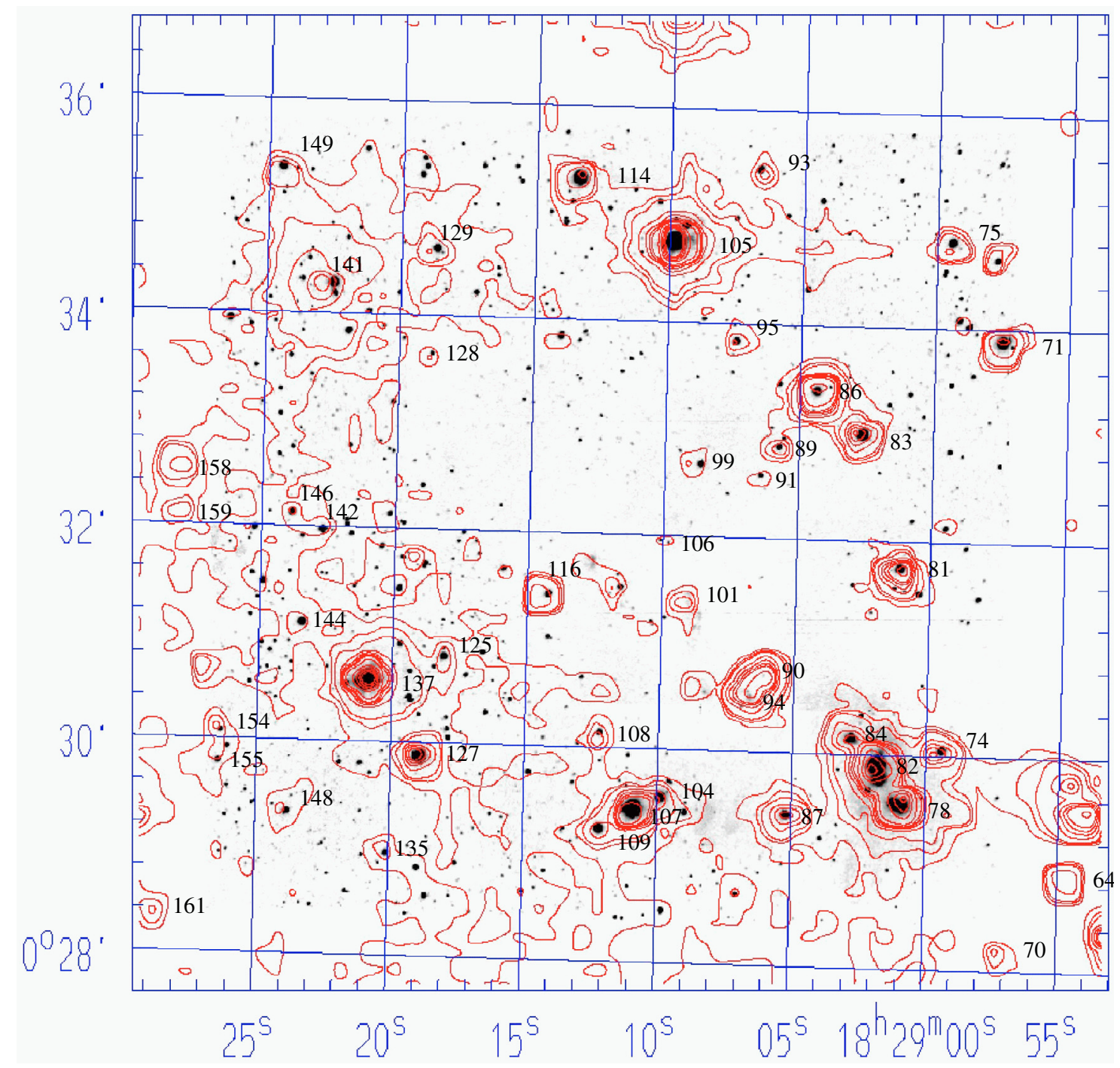

Fig. 2. Arnica/NOT $K_{\mathrm{s}}$ band $4 \times 4$ mosaic of an $8 \times 8$ arcmin part of the ISOCAM area. Contours show the ISOCAM LW2 (6.7 $\mu$ m) mosaic and ISO ID numbers are labelled (to the right of the source).

subtracts the dark current. Target images are shifted and added for each nodding cycle and flatfield corrected.

Our pointed observations with SIRCA gave $L^{\prime}$ band photometry of 46 sources in 12 fields. All these are listed in Table 3. The columns give SIRCA ID number, RA and Dec (J2000) positions $^{5}, L^{\prime}$ magnitude and its error, $K_{\mathrm{S}}$ magnitude and its error, 2MASS ID, Arnica/NOT $K_{\mathrm{S}}$ ID, ISO-NH3 ID, and other ID. The $K_{\mathrm{S}}$ band magnitudes are taken from 2MASS except for IDs $8,15,16,21,22$, and 34 , where they are taken from the Arnica/NOT observations listed in Table 2. Positions are from 2MASS when a 2MASS source is available, otherwise they are ISO positions or positions found from the $L^{\prime}$ band image with respect to other sources. For IDs 21 and 22 the positions are from the Arnica/NOT images.

Five repeated measurements of the standard star HD161903 over 4 nights gave a rms deviation of 0.048 mag. No correction was made for airmass, since this would be smaller than the variable extinction produced by the presence of dust from Sahara at the time of observing. The standards were relatively near to the targets, and the instrumental target magnitudes have been

5 The RA and Dec (J2000) positions of SIRCA sources are taken to be the 2MASS position. If no 2MASS counterpart is found, then the position is calculated with respect to other sources in the image. calibrated with the standard observation nearest in time. Magnitudes are calculated with the aperture photometry task phot in IRAF, using 20 pix (4.4") aperture radius for standards and 5 pix $\left(1.1^{\prime \prime}\right)$ for program stars. For the small aperture an aperture correction was applied to compensate the loss of flux in the wings. The correction was determined individually in each image, apart from a few cases where this was not possible and the standards were used instead. The field centred on Ser/G3-G6 is shown in Fig. 3.

\section{5. $\mathrm{H}_{2}$ line imaging with NOTCam at the NOT}

The Nordic Optical Telescope near-IR Camera/spectrograph (NOTCam $)^{6}$ was used to do narrow-band imaging in the $\mathrm{H}_{2}$ line (S (1) $1-0$ at $2.122 \mu \mathrm{m})$ and a nearby continuum $\left(\lambda_{\mathrm{c}}=2.087\right.$ and $F W H M=0.02 \mu \mathrm{m})$ at the location of the $\mathrm{NH}_{3}$ core to the northeast of the Ser/G3-G6 group. The detector was the $1024 \times 1024 \times 18 \mu \mathrm{m}$ Hawaii engineering grade array. Ser/G3-G6NE was observed on 28 May 2003. The wide field camera $\left(0.235^{\prime \prime} /\right.$ pix $)$ was used, and the observations were performed with a ramp-sampling readout mode. We integrated for

\footnotetext{
${ }^{6}$ See Abbott et al. (2000) and the URL http:// www.not.iac.es:/instruments/notcam/ for more information on NOTCam.
} 


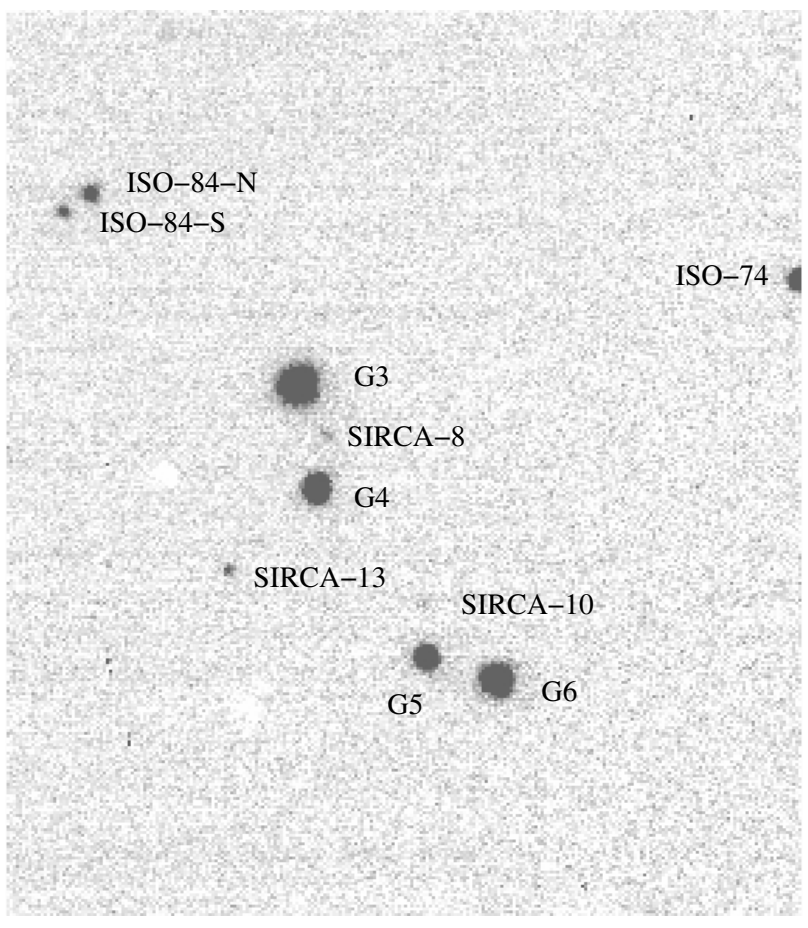

Fig. 3. SIRCA/NOT $L^{\prime}$ band image of a $70^{\prime \prime} \times 56^{\prime \prime}$ area around the Ser/G3-G6 group. North is up and East to the left.

a total of $100 \mathrm{~s}$ per sky position, reading the array every $10 \mathrm{~s}$ and using the linear regression result of these 10 readouts to lower the read noise. Altogether 20 dithered sky positions were observed, giving a total of $2000 \mathrm{~s}$ on-source for each of the two filters.

Flat fields were observed on the twilight sky creating bright faint pairs for a proper subtraction of the thermal, dark, and stray light contributions. Figure 13 shows the $\mathrm{H}_{2}$ line $(2.122 \mu \mathrm{m})$ image of the Ser/G3-G6NE region, and Fig. 14 shows the narrowband continuum $\left(\lambda_{\mathrm{c}} 2.087 \mu \mathrm{m}\right)$ image of the same region. The images suffer from some stripy pattern due to pick-up noise in the electronics at the time of observing. However, it is clear that most of the extended emission is pure $\mathrm{H}_{2}$ line emission. Only around the position of ISO-94 and ISO-101 (cf. Fig. 16) faint, extended continuum emission is seen.

The $\mathrm{H}_{2}$ line image is flux calibrated using the near-IR standards AS33-0 and AS33-1 (Hunt et al. 1998). The zeropoint difference between the $\mathrm{H}_{2}$ and the $K$ filter was found to be $2.635 \mathrm{mag}$. We transform to monochromatic flux through the relation $F_{v}=710 \times 10^{-0.4 \times m}(\mathrm{Jy})$ for a zero th magnitude star at $2.122 \mu \mathrm{m}$, assuming the stars' magnitudes are the same at 2.122 as at $2.2 \mu \mathrm{m}$. From the $\mathrm{H}_{2}$ line image of the standards, we get a conversion factor of $15.75 \mu \mathrm{Jy}$ per ADU/s for the image. The intensities in the knots range from 2 to $46 \mu \mathrm{Jy} /$ pix, and the noise in the background is $\sigma_{\text {sky }}=0.5 \mu \mathrm{Jy} / \mathrm{pix}$.

\subsection{IRAM $30 \mathrm{~m}$ telescope observations}

A $1.3 \mathrm{~mm}$ dust continuum mosaic of part of the ISOCAM coverage was taken with the IRAM 30-m telescope equipped with the MPIfR 37-channel bolometer array MAMBO-I (Kreysa et al. 1998) during four nights of observing sessions in March 1998. The passband of the MAMBO bolometer array has an equivalent width $\approx 70 \mathrm{GHz}$ and is centred at $v_{\mathrm{eff}} \approx 240 \mathrm{GHz}$.

The mosaic consists of 18 individual on-the-fly maps that were obtained in the dual-beam raster mode with a scanning velocity of $8^{\prime \prime} / \mathrm{s}$ and a spatial sampling of $4^{\prime \prime}$ in elevation. In this mode, the telescope continuously scans in azimuth along each mapped row while the secondary mirror wobbles in azimuth at a frequency of $2 \mathrm{~Hz}$. A wobbler throw of $45^{\prime \prime}$ or $60^{\prime \prime}$ was used. The typical azimuthal size of individual maps was $4^{\prime}$. The size of the main beam was measured to be $\sim 11^{\prime \prime}$ (HPBW) on Uranus and other strong point-like sources such as quasars. The pointing of the telescope was checked every $\sim 1 \mathrm{~h}$ using the VLA position of the strong, compact Class 0 source FIRS 1 in the Serpens Cloud Core (good to $\sim 0.1^{\prime \prime}$, Curiel et al. 1993); it was found to be accurate to better than $\sim 3^{\prime \prime}$. The zenith atmospheric optical depth, monitored by "skydips" every $\sim 2 \mathrm{~h}$, was between $\sim 0.2$ and $\sim 0.4$. Calibration was achieved through on-the-fly mapping and on-off observations of the primary calibrator Uranus (e.g., Griffin \& Orton 1993, and references therein). In addition, the Serpens secondary calibrator FIRS1, which has a $1.3 \mathrm{~mm}$ peak flux density $\sim 2.4$ Jy in an $11^{\prime \prime}$ beam, was observed before and after each map. The relative calibration was found to be good to within $\sim 10 \%$ by comparing the individual coverages of each field, while the overall absolute calibration uncertainty is estimated to be $\sim 20 \%$.

The dual-beam maps were reduced and combined with the IRAM software for bolometer-array data ("NIC"; cf. Broguière et al. 1995), which uses the EKH restoration algorithm (Emerson et al. 1979).

Figure 12 shows the IRAM data contour map with four bright sources labelled (MMS1, MMS2, MMS3, and MMS4), and Table 7 lists the individual sources with fluxes and positions.

\subsection{VLA $3.6 \mathrm{~cm}$ observations}

Radio continuum observations of three fields covering all four millimeter sources (MMS1-4) were made at $3.6 \mathrm{~cm}$ (central frequency $8.46 \mathrm{GHz}$, total bandwidth $100 \mathrm{MHz}$ ) with the $\mathrm{NRAO}^{7}$ Very Large Array (VLA) in a mixed B/C configuration on 22 June 2001. The on-source integration time per field was $\sim 75 \mathrm{~min}$. The amplitude calibrator was 3C286 and the phase calibrator was $1801+010$. The data were edited and calibrated using standard VLA procedures and the corresponding map cleaned using the AIPS task IMAGR with natural weighting. The synthesised beam size was $2.8^{\prime \prime} \times 2.3^{\prime \prime}(\mathrm{HPBW})$ and the primary beam (or field of view) of each observation was 5.3' (FWHM). The rms noise in each field was $\sim 16 \mu \mathrm{Jy} /$ beam, implying a $5 \sigma$ detection threshold of $\sim 0.08 \mathrm{mJy} /$ beam in the inner parts of the fields. A total of eight point-like radio sources were detected above the $5 \sigma$ level within the primary beams of the three observed VLA fields. Table 8 lists their positions (accurate to $\pm 0.5^{\prime \prime}$ ) and their $3.6 \mathrm{~cm}$ flux densities corrected for primary beam attenuation. Five VLA sources appear to be closely associated with infrared and/or millimeter sources (cf. Table 8). The other sources are probably background extragalactic sources. In addition to these robust VLA detections, we have also included a weak, tentative $(\sim 3.3 \sigma)$ radio source in Table 8 as it coincides within $1.5^{\prime \prime}$ with the $1.3 \mathrm{~mm}$ continuum source MMS1-c.

\section{IR-excess sources}

The results from the different datasets on the ISOCAM selected sample are summarised in Table 1, which is available at the CDS

\footnotetext{
7 The National Radio Astronomy Observatory is operated by Associated Universities, Inc., under a cooperative agreement with the National Science Foundation.
} 


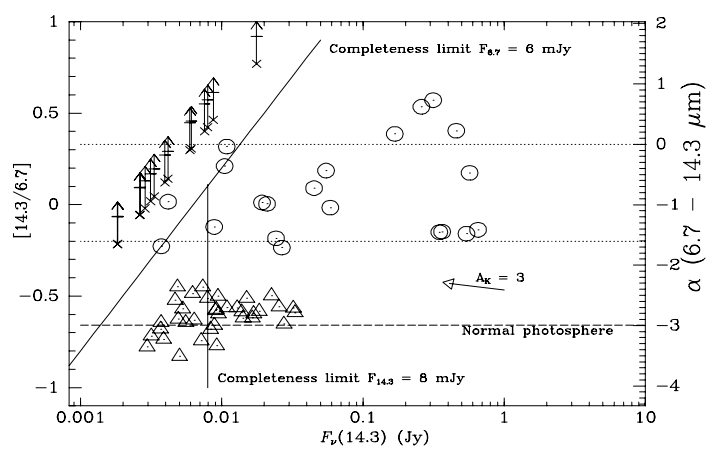

Fig. 4. The colour index [14.3/6.7], defined as $\log \left(\left(F_{v}(14.3 \mu) /\right.\right.$ $F_{v}(6.7 \mu)$ ), is shown on the $y$-axis and the flux at $14.3 \mu \mathrm{m}$ (in Jy) is shown on the $x$-axis. Sources without IR excess tend to line up around [14.3/6.7] $=-0.66$ or $\alpha=-3$ (dashed line). The 21 sources with midIR excesses (circles) are spread out over a large range in brightness. These are all YSOs. Lower limits on colour are given for sources detected at $14.3 \mu \mathrm{m}$ only (small crosses).

in electronic form only. Its first column lists the ISO identification number (ISO-nh3-\#), then the ISOCAM positions (RA and Dec in J2000 epoch) in columns two and three. Columns four and five give the Arnica $K_{\mathrm{S}}$ band magnitude and error $\left(K_{\mathrm{S}}(96)\right.$ and $\left.\sigma_{K}\right)$. Columns six to nine list the ISOCAM fluxes with uncertainties in mJy $\left(F_{v}(6.7 \mu), \sigma_{6.7}, F_{v}(14.3 \mu), \sigma_{14.3}\right)$. Columns 10 to 13 show the ISOCAM detection and photometry flag, the name of the 2MASS counterpart, the id of the NOT (Arnica $K_{\mathrm{S}}(96)$ ) counterpart, and at last a column with possible other identification. We refer to the 2MASS catalogue for further information on the 2MASS counterparts.

In the following subsections we will use various colour-magnitude and colour-colour diagrams to extract YSO candidates based on IR excess. The strategy is as follows:

- select IR-excess sources from the ISOCAM data;

- compare with the $J-H / H-K_{\mathrm{S}}$ diagram from 2MASS and search for more candidates;

- combine bands of ISOCAM and 2MASS to search for additional IR-excess sources in the $H-K_{\mathrm{S}} / K_{\mathrm{S}}-m_{7}$ diagram;

- combine SIRCA $L^{\prime}$ band with 2MASS $H$ and $K_{\mathrm{S}}$ to search for more candidates.

\subsection{The ISOCAM colour-magnitude diagram}

For the ISOCAM sources we present a colour-magnitude diagram in Fig. 4. The colour index [14.3/6.7], defined as $\log \left(F_{v}(14.3 \mu \mathrm{m}) / F_{v}(6.7 \mu \mathrm{m})\right)$, is plotted against the flux in the $14.3 \mu \mathrm{m}$ filter (magnitude indicated on upper $x$-axis). This colour index can be related to the spectral energy distribution (SED) index $\alpha=-\left(\operatorname{d} \log \lambda F_{\lambda}\right) /(\operatorname{dlog} \lambda)$ calculated between 6.7 and $14.3 \mu \mathrm{m}$, which is indicated on the right-hand $y$-axis. As seen in all star formation regions surveyed with ISOCAM, the sources tend to separate into two distinct groups: sources for which the colour index follows that of normal stellar photospheres, located around [14.3/6.7] $=-0.69$ or $\alpha=-3$, and indicated with a dashed line in Fig. 4, and sources with intrinsic IR excess. Among the 57 sources with fluxes in both bands, 21 have mid-IR excesses. These are all YSO candidates. We note that they occupy a broad range in brightness (4-900 mJy at $6.7 \mu \mathrm{m}$ ). In addition, their amount of IR excess at $14.3 \mu \mathrm{m}$ is apparently independent of brightness.

There are 16 sources detected at $14.3 \mu \mathrm{m}$ only and plotted as upper limits (small crosses) in Fig. 4. Only 8 of these

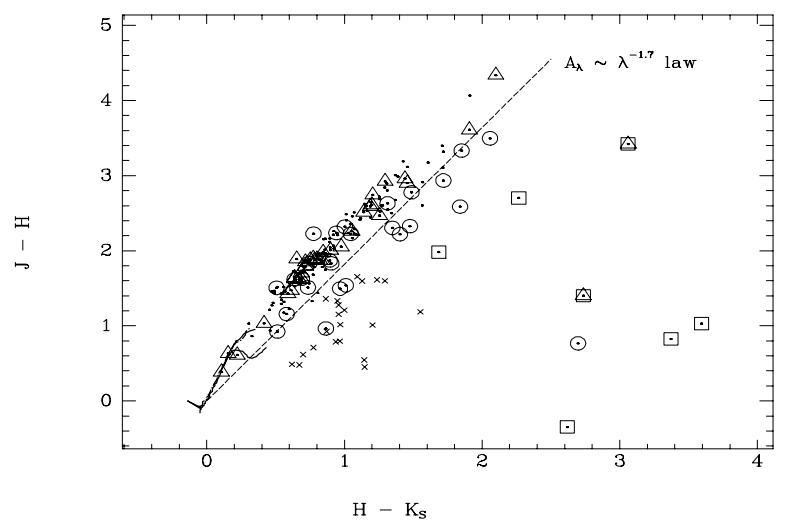

Fig. 5. The $J-H / H-K_{\mathrm{S}}$ diagram for the ISOCAM sources with 2MASS counterparts (dots). The red ISOCAM sources (open circles) merge together with the blue ISOCAM sources (open triangles) along the reddening band of normal stars. Seven ISOCAM sources appear to have excess emission in the $H-K_{\mathrm{S}}$ index (open squares), but they are all flagged 2MASS sources. Twenty faint 2MASS sources (crosses) with apparent IR excesses are discussed in the text.

have 2MASS counterparts, all with good photometric quality in $K$. The SED index taken between 2 and $14 \mu \mathrm{m}$ (cf. Sect. 4) gives $-1.01<\alpha_{\mathrm{IR}}^{2-14}<1.2$, values corresponding to an amount of IR excess typical of Class I and II types of YSOs. These are: ISO-60, 136, 143, 153, 166, 175, 181, and 182, of which ISO-143 is extremely red and outside of the plotted region in Fig. 4. We add these 8 to our list of YSO candidates. The remaining 8 might be spurious sources.

There are as many as 103 sources detected at $6.7 \mu \mathrm{m}$ only. For 97 there is a 2MASS counterpart, and all but 6 of these have good photometric quality in all three bands. These sources are discussed in Sect. 3.3.

\subsection{The $2 M A S S J-H / H-K_{S}$ diagram}

Figure 5 shows the $J-H / H-K_{\mathrm{S}}$ diagram for all the ISOCAM sources with 2MASS counterparts (dots). The blue ISOCAM sources are marked with open triangles. The red ISOCAM sources found to be YSO candidates in the previous section are marked with open circles. The loci of giant, supergiant, and main-sequence stars (Koornneef 1983) are indicated with bold curves. We have calculated the slope outlined by the ISOCAM blue sources to be $1.82 \pm 0.05$. This should be a good indicator of the cloud extinction in this diagram, and the reddening vector for an A0 star is shown by the dashed line. For the 2MASS $J H K_{\mathrm{S}}$ filter pass bands at $\lambda \lambda 1.235,1.662$, and 2.159, respectively, this slope is in accordance with the $A_{\lambda} \propto \lambda^{-1.7}$ parametrization of the NIR extinction law (Whittet 1988). This same opacity index $\beta=1.7 \pm 0.4$ was found for Serpens in the $J$ to $H$ wavelength region by Froebrich et al. (2004), also based on 2MASS data.

Figure 5 shows that the majority of the sources are clustered along the reddening band. To distinguish IR excess sources from reddened sources in the $J-H / H-K_{\mathrm{S}}$ diagram, they must be located to the right of the reddening line by at least $1 \sigma$ of the individual uncertainties in the $H-K_{\mathrm{S}}$ index. Only 10 of the 29 mid-IR excess sources have a detectable IR excess in the near-IR, as measured from the $J-H$ and $H-K_{\mathrm{S}}$ colours. This is in line with the findings of, e.g., Kaas \& Bontemps (2000), that only half or even less of the IR-excess population found from mid-IR colours is sampled by using near-IR colours. 


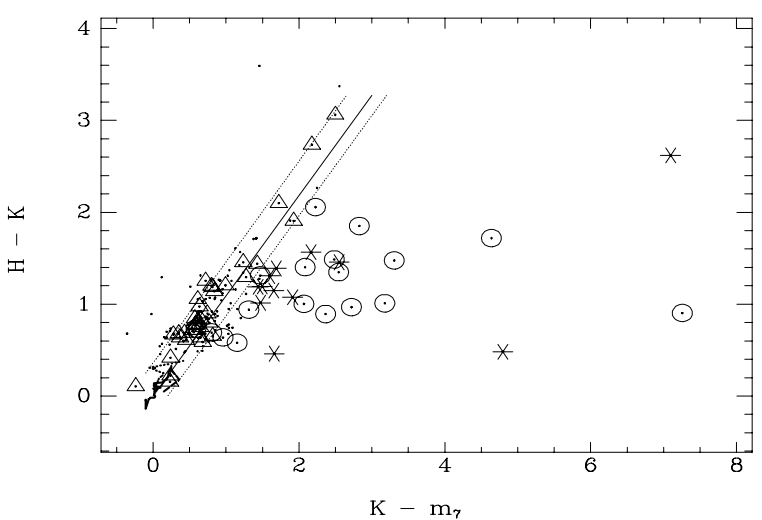

Fig. 6. The $H-K_{\mathrm{S}} / K_{\mathrm{S}}-m_{7}$ diagram for 151 ISOCAM sources. The reddening vector indicated for an $\mathrm{A} 0$ star (thin continuous line) is found by a linear fit to the blue ISOCAM sources (open triangles). The dotted lines indicate the reddening band of giants, supergiants, and mainsequence stars. The red ISOCAM sources (open circles) are practically all to the right of the reddening band. The 12 additional sources with IR excess found from this plot are marked as large asterisks.

There are 7 ISOCAM sources, for which no IR excess could be found from the ISO observations, which seem to have excesses in their $H-K_{\mathrm{S}}$ color (see Fig. 5). We note that all of these are upper limits in at least one of the 2MASS bands and should not be considered YSO candidates. Two of them are blue ISOCAM sources (ISO-59 and 116), one is independently found to have IR excess (ISO-64, see next section), but the nature of the remaining 4 cannot be assessed (ISO-31, 53, 91, 128).

In the ISOCAM field there are 1221 MASS sources with photometric quality flag ${ }^{8} \mathrm{~A}$ to $\mathrm{D}$ in all three $J H K_{\mathrm{S}}$ bands. Among these there are many faint sources not detected by ISOCAM with apparent near-IR excesses. Because these are faint sources at the survey limit, we consider those located to the right of the reddening band by at least $2 \sigma$ of the individual uncertainties in the $H-K_{\mathrm{S}}$ index. The resulting 20 sources (see Fig. 5) occupy a range of $J-H$ and $H-K_{\mathrm{S}}$ colours typical of pre-main sequence stars. As suggested by the referee, we cross-correlated these with the on-line $\mathrm{c} 2 \mathrm{~d}$ Spitzer photometry table for Serpens, and found IRAC counterparts for 19 sources. None of these have IR excess in any of the Spitzer IRAC bands, however, and therefore they cannot be proposed as bona-fide IR excess YSO candidates. The apparent excesses in $H-K_{\mathrm{S}}$ are not understood and may mostly be the result of statistical fluctuations in the 2MASS data, since none of the 20 sources is located to the right of the reddening band by more than $5 \sigma$ of the individual errors in the $H-K_{\mathrm{S}}$ index. More data is needed to establish the near-IR colours of these sources.

\subsection{The $H-K_{S} / K_{S}-m_{7}$ diagram}

As seen in Fig. 5, the $J-H / H-K_{\mathrm{S}}$ diagram does not extract nearly half of the IR-excess sources identified with ISOCAM. The IR excess does not separate well from the reddening in the $J-H$ and $H-K_{\mathrm{S}}$ colours, and the blue and red ISOCAM sources tend to merge together along the reddening band. As shown in, e.g., Kaas et al. (2004), the $H-K_{\mathrm{S}} / K_{\mathrm{S}}-m_{7}$ diagram better distinguishes IR excess from reddening. By using this diagram we can study the 103 ISOCAM sources for which there are $6.7 \mu \mathrm{m}$

\footnotetext{
8 See the 2MASS catalogue description at http://www.ipac.caltech.edu/2mass/releases/allsky/doc/ for details.
}

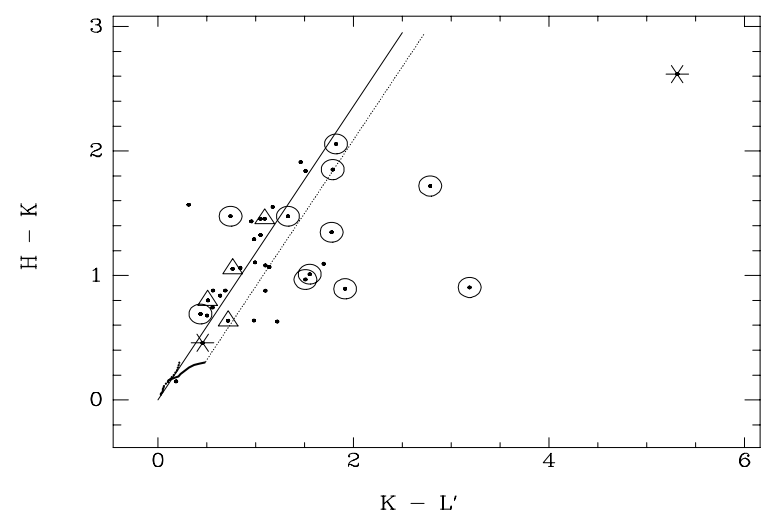

Fig. 7. The $H-K_{\mathrm{S}} / K_{\mathrm{S}}-L^{\prime}$ diagram for 39 sources (dots). We show by overplotting symbols the red ISOCAM sources (open circles), blue ISOCAM sources (open triangles), and the additional IR-excess sources identified in the $H-K_{\mathrm{S}} / K_{\mathrm{S}}-m_{7}$ diagram (large asterisks). The reddening band of normal stars is indicated (dotted line) as is the reddening line for an A0 star (thin continuous line). Four new IR excess sources are identified.

fluxes only (see Fig. 6). The reddening vector indicated is empirically found from this dataset. The slope $1.09 \pm 0.01$ is found by a linear fit to the blue ISOCAM sources. For comparison, the slope was 1.23 for the Serpens Cloud Core (Kaas et al. 2004). The width of the reddening band of giants, super giants and main-sequence stars, whose intrinsic colours are outlined by the bold face curves, is indicated. The red ISOCAM sources are all, except two (132 and 167), found to the right of the reddening band. We find a total of 26 IR-excess objects from the $H-K_{\mathrm{S}} / K_{\mathrm{S}}-m_{7}$ diagram, i.e., those who are located to the right of the reddening band of normal stars by more than $1 \sigma$ in the $K_{\mathrm{S}}-m_{7}$ colour index. Twelve of these are new, i.e., not selected in the colour diagrams discussed above.

\subsection{The $H-K_{S} / K_{S}-L$ diagram}

The $H-K_{\mathrm{S}} / K_{\mathrm{S}}-L^{\prime}$ diagram is shown in Fig. 7. We plot the 39 sources available in these colours, and overplot red ISOCAM sources, blue ISOCAM sources, and additional IR-excess sources found from the $H-K_{\mathrm{S}} / K_{\mathrm{S}}-m_{7}$ diagram. The intrinsic colours of main sequence and giant stars (Bessell \& Brett 1988) are indicated, too. The reddening vector of an A0 star is drawn applying the $A_{\lambda} \propto \lambda^{-1.7}$ law (Whittet 1988) again, giving a slope of 1.18. The reddening band for normal stars is also indicated, and IR excess sources must be located to the right of this line by more than one $\sigma$ of the individual errors in the $K_{\mathrm{S}}-L^{\prime}$ colours. We note that the distinction between the red and blue ISOCAM sources is not so clear in this diagram as in the $H-K_{\mathrm{S}} / K_{\mathrm{S}}-m_{7}$ diagram, but significantly clearer than in the $J-H / H-K_{\mathrm{S}}$ diagram. There are no blue ISOCAM sources in the IR-excess locus, but there are five red ISOCAM sources in the locus of reddened normal stars. In total 11 sources have IR excess in the $K_{\mathrm{S}}-L^{\prime}$ colour, of which four were not previously identified as IR-excess objects. None of these four is in the ISOCAM selected sample of Table 1, but two of them are the objects G4 and G5, which are very close to G3 (ISO-82) and G6 (ISO-78), respectively. The third object is in the same area just SE of G4, and the fourth object is a faint star in the SIRCA field of ISO-167. 


\subsection{Extracted YSO candidates}

From the 186 ISOCAM detections we have extracted 29 YSO candidates from the ISOCAM data, and 12 additional from the combination of ISOCAM and 2MASS using the $H-K_{\mathrm{S}} / K_{\mathrm{S}}-m_{7}$ diagram. This gives a total sample of 41 IR-excess YSOs within the ISOCAM selected sample. In addition, we find 4 IR-excess objects from the $H-K_{\mathrm{S}} / K_{\mathrm{S}}-L^{\prime}$ diagram. Summing up, this gives a total of 45 YSOs.

\section{The YSO population}

According to the current empirical picture of young stellar evolution, low mass YSOs go through evolutionary phases with different Spectral Energy Distribution (SED) characteristics: Class 0, Class I, (flat-spectrum, ) Class II, and Class III sources (Adams et al. 1987; Lada 1987; André et al. 1993; André \& Montmerle 1994).

The Class 0 sources are in the deeply embedded main accretion phase and have circumstellar envelope masses larger than their central stellar mass. The defining characteristics of a Class 0 is: strong, centrally condensed dust continuum emission in the submm, very little emission shortward of $10 \mu \mathrm{m}$, and powerful jet-like outflows (André et al. 2000). Thus, we do not expect to sample these sources in the mid-IR, although ISOCAM did detect a few Class 0 sources, and Spitzer with its improved sensitivity has detected many more. The dust continuum mapping at $1.3 \mathrm{~mm}$ with IRAM should sample the Class 0 sources, and we refer to Sect. 6 for a discussion of these results. The Class I, flat-spectrum, and Class II sources, however, have IR SEDs that are reasonably well sampled by flux measurements at $2.2,6.7$, and $14.3 \mu \mathrm{m}$.

\subsection{Classifying the IR-excess sources}

We have calculated the SED index between 2.2 and $14.3 \mu \mathrm{m}$, $\alpha_{\mathrm{IR}}^{2-14}$, which is close to the index used by Lada \& Wilking (1984) and Lada (1987) to define the three classes, I, II, and III, originally. We use the updated IR spectral classification scheme (André \& Montmerle 1994; Greene et al. 1994) and define YSOs with $\alpha_{\mathrm{IR}}^{2-14}>0.3$ as Class I sources, YSOs with $\alpha_{\mathrm{IR}}^{2-14}$ in the interval $[-0.3,0.3]$ as flat-spectrum sources, and YSOs with $\alpha_{\mathrm{IR}}^{2-14}$ in the interval $[-1.6,-0.3]$ as Class II sources. IR-excess sources with $\alpha_{\mathrm{IR}}^{2-14}<-1.6$ are tentatively defined as Class III sources. We note that because our YSO sample is selected from IR excesses most Class III objects are missed. Only a few with a small detectable IR excess are captured, probably those objects in transition between classes II and III. Class III sources with no IR excess cannot be distinguished from field stars using our data. Additional tools such as X-ray surveys must be applied to sample the Class III population.

Figure 8 shows the $\alpha_{\mathrm{IR}}^{2-7}$ index versus the $\alpha_{\mathrm{IR}}^{2-14}$ index with the corresponding $K-m_{7}$ and $K-m_{14}$ colours in magnitudes given on the opposite axes. The loci of the different YSO classes are indicated. Sources with no IR excess line up in the lower left of the diagram along the extinction vector. The vector is taken from Kaas et al. (2004) and has a length corresponding to $\sim 60$ mag of visual extinction. Most sources are found with $A_{V}$ about 10-20 mag, while one object (ISO-88 = HD 170377) has no extinction and is perhaps a foreground star or a Class III cluster member at the front side of the cloud. According to SIMBAD it is a bright $V=8.96$ mag optical star with spectral type $\mathrm{K} 2$. Sources with IR excess mainly occupy the Class II locus, while there are also a few in the Class I, flat-spectrum, and Class III

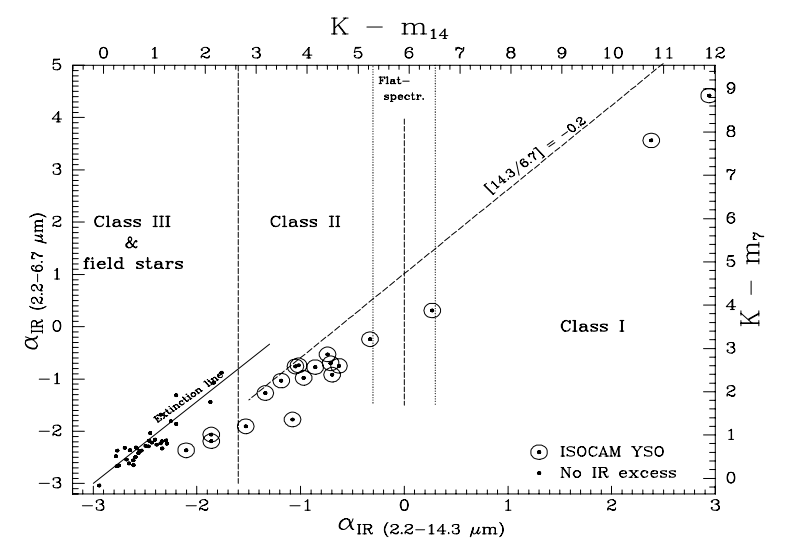

Fig. 8. The spectral index $\alpha_{\mathrm{IR}}^{2-7}$ versus $\alpha_{\mathrm{IR}}^{2-14}$ or equivalently, the $K-m_{7} / K-m_{14}$ colour diagram. The locations of Class I, Class II, and Class III sources and field stars are indicated, as well as the flatspectrum source locus.

loci. Compared to the Serpens Cloud Core (Kaas et al. 2004) this region has fewer Class I and flat-spectrum objects. The reddest Class I, however, has $\alpha_{\mathrm{IR}}^{2-14}=2.94$, which is redder than any of the Class Is in the Serpens Cloud Core. Both very red objects (ISO-90 and ISO-94) are located in a core of active star formation near the dense mm core MMS2 (see Sects. 6.1 and 6.2). At least for one of these (ISO-94) the emission in the $K$-band is dominated by line emission.

We note the almost linear relationship between the two SED indices $\alpha_{\mathrm{IR}}^{2-7}$ and $\alpha_{\mathrm{IR}}^{2-14}$ in Fig. 8. As in Kaas et al. (2004) we use this relationship to classify objects from the $\alpha_{\mathrm{IR}}^{2-7}$ index only, allowing us to classify objects not detected at $14.3 \mu \mathrm{m}$. From Fig. 8 we see that the colour [14.3/6.7] is essential to distinguish Class III objects with IR-excess from reddened stars. It is also evident that for very high extinction there is a risk of confusing Class II objects with reddened stars unless the [14.3/6.7] colour is known. Nevertheless, with this caveat in mind, we classify 12 more sources than those plotted in Fig. 8. Thus in the sample of IR-excess YSOs, we define as Class Is those that have $\alpha_{\mathrm{IR}}^{2-7}>$ $1.2\left(K-m_{7}>4.8\right)$, Class IIs those that have $\alpha_{\mathrm{IR}}^{2-7}<-0.25$ $\left(K-m_{7}<3.2\right)$, and flat-spectrum sources as those in between.

There are, however, 6 IR-excess sources with neither of the two SED indices $\left(\alpha_{\mathrm{IR}}^{2-14}\right.$ and $\left.\alpha_{\mathrm{IR}}^{2-7}\right)$ available. In the following we will classify these sources on an ad hoc basis. Four of them are SIRCA sources (id 9, 11, 13, and 44 in Table 3) with no ISOCAM detections. Plotting the various YSO classes in the $H-K / K-L^{\prime}$ diagram we see that these four are located in the region occupied by the Class II sources in our sample. Therefore we tentatively classify them as Class IIs. ISO-62 is a red ISOCAM source without a 2MASS counterpart located in the dense Ser/G3-G6SW ammonia core. Judging from the 2MASS sensitivity we estimate that ISO-62 has $\alpha_{\mathrm{IR}}^{2-14}>2$ and we tentatively classify it as a Class I object. ISO-101, located in the dense Ser/G3-G6NE ammonia core, is a red ISOCAM source not detected in the near-IR continuum. The NOTCam images show extended emission in the $\mathrm{H}_{2}$ line at $2.122 \mu \mathrm{m}$ and no emission in the continuum filter at $2.087 \mu \mathrm{m}$. Its rather blue [14.3/6.7] colour probably also reflects a $\mathrm{H}_{2}$ line emission (cf. Fig. 13). Some faint extended emission in the $L^{\prime}$ band is barely detected at this position (see Table 3). The lower limit estimate of $\alpha_{\mathrm{IR}}^{2-14}$ first suggested that ISO-101 could be a Class I or flat-spectrum source, but considering the above discussion we do not count this object as a continuum source (see discussion in Sects. 4.1 and 6.1). 
Table 4. The 5 Class I and the 5 flat-spectrum sources. Cross-correlated with Spitzer YSO candidates from Harvey et al. (2006).

\begin{tabular}{rlcrr}
\hline \hline ISO & Other ID & $\alpha_{\mathrm{IR}}^{2-7}$ & $\alpha_{\mathrm{IR}}^{2-14}$ & $\Delta K$ \\
\hline 90 & IRAS 18265+0028, Spitzer-16 & 4.42 & 2.94 & n.a. \\
$94^{1}$ & Spitzer-17 & 3.56 & 2.38 & 0.64 \\
$62^{2}$ & & $>3$ & $>2$ & n.a. \\
$143^{3}$ & & & 1.20 & n.a. \\
64 & Spitzer-5 & 2.93 & & n.a. \\
\hline 86 & & 0.31 & 0.27 & -0.74 \\
60 & Spitzer-3 & & 0.02 & n.a. \\
182 & & & -0.04 & n.a. \\
153 & & & -0.14 & n.a. \\
121 & & 1.06 & & n.a. \\
\hline
\end{tabular}

${ }^{1} \mathrm{H}_{2}$ line emission probably dominates the $K$ band flux. ${ }^{2}$ Lower limits on $\alpha_{\mathrm{IR}}^{2-14}$, but $\alpha_{\mathrm{IR}}^{4-14}=1.54 .{ }^{3}$ Not located within the $1.3 \mathrm{~mm}$ emission, and its Class I nature is questionable.

In total, the IR-excess population we have found in the Ser/G3-G6 complex consists of 5 Class I sources, 5 flat-spectrum sources, and 31 Class II sources. In addition, we see 3 Class IIIs with some small IR-excesses, noting that this class is not sampled by our IR-excess selection criterion.

The Class I/Class II number ratio of $5 / 31$ is more or less typical for star-forming regions and compares with $16 / 123$ in $\rho$ Ophiuchi (Bontemps et al. 2001) and 5/42 in Chamaeleon I (Kaas 1999b). This is in strong contrast to the large fraction of Class I sources found in the Serpens Cloud Core (Kaas et al. 2004).

\subsection{Class I and flat-spectrum candidates}

There is a total of 10 IR-excess protostar candidates listed in Table 4: 5 Class Is and 5 flat-spectrum sources. We note that the classification is tentative in the sense that only lower limits on the SED indices can be given in the case of ISO-62. Also, the indices of ISO-94 are lower limits since the $K$-band flux is dominated by $\mathrm{H}_{2}$ line emission. These sources may be even younger than we have indicated. Nevertheless, in the following we will use our tentative classification.

Two of the Class Is are located in the ammonia core Ser/G3-G6NE (ISO-90 and 94), and another two (ISO-62 and 64) in the core Ser/G3-G6SW. As shown in the IRAM $1.3 \mathrm{~mm}$ continuum map in Fig. 12, all but one Class Is are located in the high density regions outlined by the $1.3 \mathrm{~mm}$ continuum emission. The exception, ISO-143, lies to the NE of the two ammonia cores in the vicinity of ISO-141, It is extended in both $I S O$ bands and not detected as a point source at $6.7 \mu \mathrm{m}$ (therefore not visible in Fig. 2). Its Class I nature might be questionable. The flat-spectrum sources are partly located inside the cores and partly outside.

\subsection{The Class II and Class III sources}

Our sample of pre-main sequence stars in the Ser/G3-G6 complex comprises the Class II objects listed in Table 5 and the few Class IIIs our selection method is capable of seeing, listed in Table 6. As emphasized in Sect. 4.1, our YSO selection criterion is IR excess, and therefore we do not sample the Class III population except for occasional transition objects between Class II and III.

As shown in Fig. 12 the spatial distribution of the Class II sources is relatively scattered, apart from the small cluster of
Table 5. The 31 Class II sources. The cross-correlated Spitzer YSO candidates from Harvey et al. (2006) are indicated in the last column.

\begin{tabular}{rrrrl}
\hline \hline ISO & $M_{J}$ & $A_{J}$ & $\Delta K$ & Other ID \\
\hline 7 & 4.72 & 3.51 & n.a. & \\
26 & 5.67 & 4.44 & n.a. & \\
74 & 3.50 & 4.88 & 0.18 & \\
78 & 3.06 & 1.74 & 0.05 & CoKu-Ser-G6, NOT-158 \\
80 & 6.06 & 2.58 & n.a. & SIRCA-5,Spitzer-10 \\
81 & 2.80 & 6.28 & 0.04 & SIRCA-7,NOT-162,Spitzer-11 \\
82 & 2.31 & 1.63 & -0.11 & CoKu Ser G3, NOT-201 \\
$84^{1}$ & 5.03 & 3.74 & -0.72 & SIRCA-15 and 16, Spitzer-14 \\
96 & 4.76 & 3.68 & n.a. & SIRCA-23 \\
100 & 3.90 & 5.73 & n.a. & SIRCA-28 \\
102 & 5.67 & 3.64 & n.a. & \\
103 & -0.76 & 6.70 & n.a. & SIRCA-30 \\
105 & 0.55 & 3.47 & 0.50 & \\
122 & 3.41 & 4.03 & n.a. & \\
136 & 7.00 & 4.39 & n.a. & \\
137 & -0.28 & 4.51 & 0.49 & Spitzer-21 \\
139 & 2.42 & 5.41 & n.a. & \\
140 & 4.28 & 4.49 & n.a. & \\
141 & 4.43 & 0.22 & -0.07 & SIRCA-41,NOT-898 \\
147 & 3.80 & 4.55 & n.a. & \\
154 & 5.70 & 3.72 & 0.25 & Spitzer-23 \\
156 & 4.78 & 3.94 & n.a. & \\
166 & 6.53 & 3.48 & n.a. & Spitzer-24 \\
175 & 9.54 & 0.19 & n.a. & \\
176 & 4.46 & 0.78 & n.a. & \\
178 & 3.40 & 5.18 & n.a. & \\
181 & 6.32 & 2.49 & n.a. & \\
- & 4.26 & 2.04 & -0.28 & CoKu Ser G5, Spitzer-12 \\
- & 3.23 & 1.18 & -0.10 & CoKu Ser G4 \\
- & 7.10 & 0.79 & -0.14 & SIRCA-13 \\
- & 5.40 & 3.97 & n.a. & SIRCA-44 \\
\hline & & & & \\
\hline
\end{tabular}

1 Double source resolved by SIRCA and NOT/Arnica (NOT-239 and 247), but not resolved by ISO and 2MASS.

Class IIs at the location of CoKu-Ser/G3-G6. We note that all four objects CoKu-Ser/G3-G6, earlier found to be T Tauri sources with strong $\mathrm{H} \alpha$ emission (Cohen \& Kuhi 1979) are independently classified here as Class II sources. For the Class II sample we present $M_{J}$ and $A_{J}$ as calculated in Sect. 5.1 in Table 5. The fourth column represents the variation in the $K_{\mathrm{S}}$ magnitude between the 2MASS data (Jul. 2000) and the Arnica/NOT (Aug. 1996) dataset in the sense $K_{\mathrm{S}}(1996)$ $K_{\mathrm{S}}(2000)$. Among the 12 Class IIs where $K_{\mathrm{S}}$ band photometry is available for both epochs, as many as 6 (or 50\%) have varied by more than $\Delta K=0.2 \mathrm{mag}$.

\subsection{Comparison with recent Spitzer results}

At the finalization of this paper we became aware of the recent publication of Harvey et al. (2006) who list Spitzer mid-IR fluxes for 24 YSO candidates found in a $12^{\prime} \times 12^{\prime}$ area centred on the Ser/G3-G6 complex, which they refer to as Serpens Cluster B. We have cross-correlated these with our results and find that we have 4 Class I and 7 Class II sources in common, while Harvey et al. (2006) classify one blue ISOCAM source as a Class II and find 11 YSO candidates that go undetected by ISOCAM. The Spitzer source number is added under Other ID in both Tables 4 and 5. Comparing the SED indices with the Spitzer results, the classification is in agreement for all but one source, ISO-64 (Spitzer-5), where the Spitzer data suggests it is a Class II. The ISOCAM $6.7 \mu \mathrm{m}$ flux is about twice as high as the interpolated 
Table 6. The 3 Class III sources with some IR-excess. Note that our study does not sample the Class III sources.

\begin{tabular}{rcccrrr}
\hline \hline ISO & $\alpha_{\mathrm{IR}}^{2-7}$ & $\alpha_{\mathrm{IR}}^{2-14}$ & $J-H$ & $H-K$ & $K-L^{\prime}$ & $\Delta K$ \\
\hline 2 & -2.06 & -1.86 & 1.619 & 0.638 & n.a. & n.a. \\
132 & -2.36 & -2.10 & 1.511 & 0.735 & n.a. & n.a. \\
167 & -2.19 & -1.86 & 1.628 & 0.691 & 0.435 & n.a. \\
\hline
\end{tabular}

value between the Spitzer bands at 5.8 and $8.0 \mu \mathrm{m}$. This could be due to strong $\mathrm{H}_{2}$ line emission in the $6.7 \mu \mathrm{m}$ band or also to source variability.

We also note that both ISO-62 and ISO-101 are clearly detected in the IRAC images, but not listed among the Spitzer YSO candidates of Harvey et al. (2006), probably because of their extended nature.

\section{Luminosity and mass function}

\subsection{Stellar luminosities}

We do not attempt to estimate luminosities and masses for the protostar candidates (flat-spectrum and Class I sources). In this section we concentrate on the Class II sources. It is probably justified to assume that our Class II sample in Table 5 is dominated by Classical T Tauri Stars (CTTS), since there are only very few examples of weak-lined T Tauri Stars (WTTS) with the amount of IR excess compatible with a Class II designation.

To estimate the reddening of each object we assume an intrinsic colour of $(J-H)_{0}=0.85$, i.e., the median value found for CTTS (Strom et al. 1993; Meyer et al. 1997). The extinction in the $J$-band is then found from the relation $A_{J}=2.53 \times$ $\left[(J-H)-(J-H)_{0}\right]$, obtained from the $A_{\lambda} \propto \lambda^{-1.7}$ extinction law (cf. Sect. 3.2). The absolute $J$-band magnitudes are $M_{J}=m_{J}-A_{J}-D M$, where the distance modulus is $D M=6.76$ for the distance of 225 pc (Straižys et al. 2003). Table 5 lists the Class II YSOs with extinction and absolute $J$-band magnitudes.

Apart from the photometric uncertainty in $J$, which is $\pm 0.05 \mathrm{mag}$ on the average, the uncertainties introduced in $M_{J}$ arise from 1) the spread in the intrinsic $(J-H)_{0}$ colour of our targets, 2) the uncertainty in the distance estimate, and 3 ) the amount of veiling in the $J$-band. The spread around the median value of $(J-H)_{0}$ for CTTS is of the order of \pm 0.15 , which translates directly into $\pm 0.38 \mathrm{mag}$ in the estimate of $A_{J}$. The quoted uncertainty in the distance of \pm 55 pc (Straižys et al. 2003) gives an error in the distance modulus of ${ }_{-0.61}^{+0.48}$ mag. The average amount of $J$-band veiling for 22 CTTS in Taurus-Auriga was found by Folha \& Emerson (1999) to be as high as $r=0.57$. About $44 \%$ of the CTTS have this or a higher amount of veiling. This means that in nearly half of the cases, as much as $36 \%$ or more of the flux in the $J$-band $(r /(1+r))$ is non-photospheric. The errors introduced by not correcting for the veiling can thus be of the order of $0.4 \mathrm{mag}$ in the direction of overestimating the source brightness. On the assumption that these sources of errors are independent, we have a total uncertainty (added in quadrature) in the estimate of $M_{J}$ of ${ }_{-0.84}^{+0.63} \mathrm{mag}$.

The $M_{J}$ luminosity function of the pre-main sequence sample is shown in Fig. 9. A binsize of $0.8 \mathrm{mag}$ was chosen to account for the possible uncertainties in the $M_{J}$ estimates discussed above. The vertical lines indicate the substellar limit for the two extreme assumptions on age: $1 \mathrm{Myr}$ and $10 \mathrm{Myr}^{9}$.

9 The age of CTTS is currently believed to be of the order of a few Myr, but there is some evidence of longlived $\sim 10$ Myr old circumstellar disks (Lawson et al. 2002; Lyo et al. 2003).

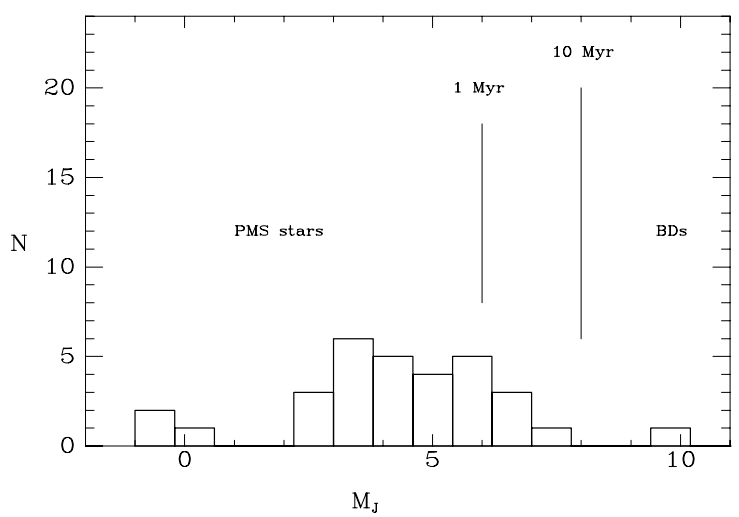

Fig. 9. The $M_{J}$ luminosity function for the Class II sources The range of young BDs and young stars is indicated by the substellar limits (vertical lines) for the two ages 1 and $10 \mathrm{Myr}$.

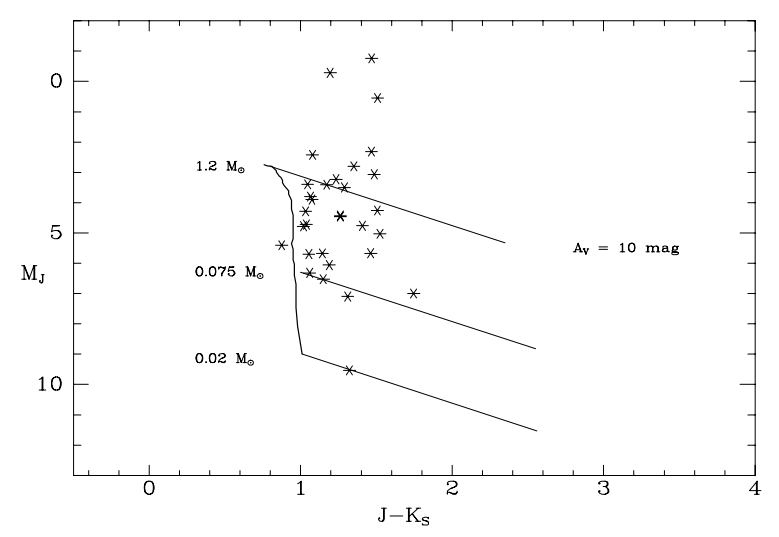

Fig. 10. The $M_{J}$ vs. $J-K_{\mathrm{S}}$ colour magnitude diagram for the Class II sources. The $2 \mathrm{Myr}$ isochrone of Baraffe et al. (1998) and reddening vectors of length $A_{V}=10 \mathrm{mag}$ are drawn. Note that the $J-K_{\mathrm{S}}$ colour is dereddened according to the dereddening scheme explained in the text.

The $M_{J}$ distribution is relatively flat over the whole sampled range. The completeness limit of the Class II sample is based on the completeness limit of the mid-IR survey, which is $6 \mathrm{mJy}$ for the $6.7 \mu \mathrm{m}$ band (cf. Sect. 2.1). We have made a linear fit between the $6.7 \mu \mathrm{m}$ flux and $M_{J}$ for the 23 Class II sources that have both values. The $6 \mathrm{mJy}$ completeness limit was found to correspond to $M_{J}=5.6 \pm 0.2 \mathrm{mag}$.

In Fig. 10 we plot $M_{J}$ versus $J-K_{\mathrm{S}}$ for the 31 Class II sources. The $2 \mathrm{Myr}$ isochrone from the evolutionary models of Baraffe et al. (1998) is drawn with the reddening vectors indicated for the lowest and highest mass included in the model, as well as the substellar mass limit at $0.075 M_{\odot}$.

The $J-K_{\mathrm{S}}$ colour has been corrected for reddening as discussed above. Because of intrinsic IR-excess and possibly also insufficient dereddening in some cases, practically all sources fall to the right of the $2 \mathrm{Myr}$ isochrone. The sample comprises objects in the mass range from above $1.2 M_{\odot}$ to $0.07 M_{\odot}$ (one object reaching to $0.02 M_{\odot}$ ), assuming an age of $2 \mathrm{Myr}$.

\subsection{Candidate young brown dwarfs with disks}

The border between stars and brown dwarfs (at $\sim 0.075 M_{\odot}$ ) is indicated both in Figs. 9 and 10. We note that between 1 and 5 of the 31 Class II sources in Table 5 have substellar masses, judging from Fig. 9 and the age range 1-10 Myr. This same fraction of BDs among ISOCAM selected Class II objects (i.e., 20\% for 
ages of a few Myr) was also found in the Chamaeleon I cloud (Olofsson et al. 1998; Persi et al. 2000), the $\rho$ Ophiuchi cloud (Bontemps et al. 2001), and the Serpens Cloud Core (Kaas et al. 2004).

All the sources in our YSO samples have IR-excess, of which 31 sources are designated Class II objects. Class IIs have a midIR SED compatible with circumstellar dust distributed in a disk. The substellar objects in our study are therefore most probably young BDs with circumstellar disks. We note that since the selection criteria in our study is IR excess, we do not sample any BDs without disks.

\subsection{The Serpens Class II mass function}

The 31 Class II sources from this paper are added to the sample of 43 Class II sources in the Serpens Cloud Core region from Kaas et al. (2004) to improve the small number of statistics for the Serpens Class II luminosity function (LF). The two samples are from two different embedded young clusters separated by a projected distance of $45 \operatorname{arcmin}(\sim 3 \mathrm{pc})$, but probably located in the same cloud system and at the same distance (225 pc adopted from Straižys et al. 2003).

For the 31 Class II sources of this paper (cf. Table 5) we have calculated the stellar luminosity $L_{\star}$ from $M_{J}$ using the relation $\log L_{\star}=1.49-0.466 * M_{J}$ found by Bontemps et al. (2001). The total Serpens sample now consists of 74 Class II sources. Figure 11 shows the observed luminosity function (LF) of the combined sample of Class II sources together with model LFs calculated for co-eval formation for a set of four ages: $0.5,1,2$, and 5 Myr.

We assume as a first approximation co-eval formation of the Class II sources. Though admittedly a simplification, this assumption may be viable as we are treating a subset of cluster members constrained evolutionarily: Class II sources are currently believed to have ages of a few Myrs. Also, it can be argued that star formation may proceed in several bursts giving rise to generations of YSOs (Kaas et al. 2004).

For easy comparison we show the model LFs here calculated as in Kaas et al. (2004) using the pre-main sequence evolutionary models of D'Antona \& Mazzitelli (1998) and applying three different IMFs. Here we show the Salpeter IMF (Salpeter 1955), the Scalo three-segment power-law IMF (Scalo 1998), and the Kroupa three-segment power-law IMF (Kroupa 2001). With the IMF of the form $\mathrm{d} N \propto m^{\Gamma} \mathrm{d}(\log m)$, the Salpeter (1955) $\mathrm{IMF}^{10}$ has $\Gamma=-1.35$.

In Fig. 11 the LFs produced by these IMFs for the four ages mentioned above are shown superposed on the observed LF, i.e., as the number of sources per bin versus $\log L$. The completeness limit at $L \sim 0.08 L_{\odot}$ is also given (cf. Sect. 5.1). The normalization has been made to the number of sources above the completeness limit. The bin width in the histogram is $\operatorname{dlog} L=0.325$, based on a factor of two uncertainty in the luminosity estimate.

The increased sample in this work confirms the findings in (Kaas et al. 2004) that the Serpens Class II LF is compatible with coeval formation 2 Myr ago and an IMF of the threesegment power-law type all the way down to the completeness limit at $L \sim 0.08 L_{\odot}$, which corresponds to $M \sim 0.15 M_{\odot}$ for 2 Myr. In the plots we have extrapolated the Scalo IMF and the

10 The Scalo (1998) IMF has $\Gamma=-1.3$ for $10 M_{\odot}<m<100 M_{\odot}$, $\Gamma=-1.7$ for $1 M_{\odot}<m<10 M_{\odot}$, and $\Gamma=-0.2$ for $0.1 M_{\odot}<m<1 M_{\odot}$. The Kroupa (2001) IMF has $\Gamma=-1.3$ for $m>0.5 M_{\odot}, \Gamma=-0.3$ for $0.08 M_{\odot}<m<0.5 M_{\odot}$, and $\Gamma=0.7$ for $m<0.08 M_{\odot}$, see Bonnell et al. (2006). In Kaas et al. (2004) we instead used Kroupa et al. (1993).
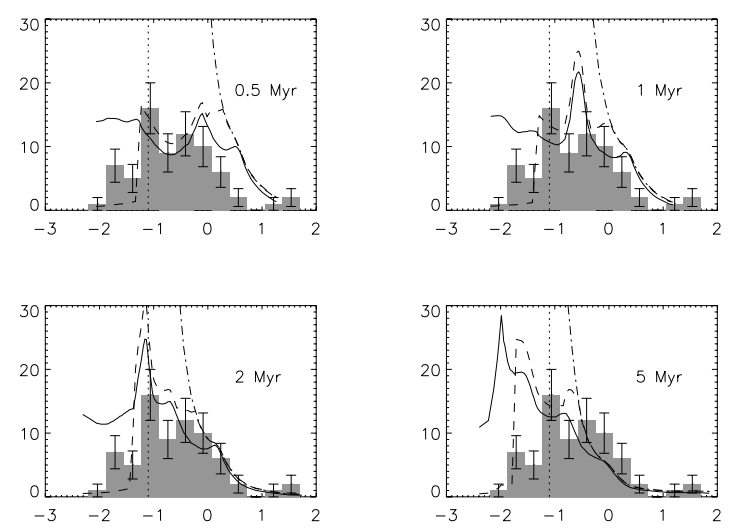

Fig. 11. The histogram shows the observed LF of Class II sources from the Ser/G3-G6 region (this paper) and the Serpens Cloud Core (Kaas et al. 2004) as the number of sources per bin vs. $\log L$. Overplotted are the synthetic LFs of a coeval population calculated on the basis of the pre-main sequence tracks of D'Antona \& Mazzitelli (1998). Both the Scalo (1998) three-segment power-law IMF (solid line) and the Kroupa (2001) three-segment power-law IMF (dashed line) trace well the observed LF for a coeval population of $\sim 2 \mathrm{Myr}$ down to the completeness limit (vertical dotted line). The Salpeter (1955) IMF (dashed-dotted line) has been included for reference.

Salpeter IMF to masses below which these IMFs are defined, i.e., beyond $0.1 M_{\odot}$ and $0.4 M_{\odot}$, respectively. We note that even the Salpeter IMF explains the data at high luminosities.

The peak in the observed LF at $L \sim 0.09 L_{\odot}$ coincides with the completeness limit and cannot be used to constrain the age. An age dependent peak in the LF is expected as a result of the piling up of stars in a given luminosity bin because of the slowing-down effect deuterium burning has on YSO contraction (Zinnecker et al. 1993). Nevertheless, Fig. 11 shows that neither of the three IMFs produce compatible LFs for ages much younger or older than 2 Myr. The model LFs for coeval populations that formed $0.5 \mathrm{Myr}$ and $1 \mathrm{Myr}$ ago have more sources than observed in the luminosity bins around $3 L_{\odot}$ and $0.3 L_{\odot}$, respectively. For ages above $2 \mathrm{Myr}$ the model LFs produce fewer sources than observed for luminosities above $0.7 L_{\odot}$. This tendency starts already at an age of $3 \mathrm{Myr}$, but for $5 \mathrm{Myr}$ the deviation is larger than the error bars for several consecutive bins in the observed LF.

We thus conclude that an age of $2 \mathrm{Myr}$ is a good fit for the Class II population, although co-eval star formation in a strict sense is unlikely. We cannot exclude models where star formation proceeds in a more continuous way. However, we find that continuous SF over intervals shorter than $2 \mathrm{Myr}$ is incompatible with the observed LF. A single burst of star formation that took place 2 Myr ago can fully explain the data. Bursts of short duration produce model LFs that resemble the co-eval LFs, while increasing the burst duration smooths out the structure in the LFs.

To distinguish between the Scalo (1998) and the Kroupa (2001) IMFs for the Serpens Class II sources we need to extend the completeness limit from $0.08 L_{\odot}$ to $0.04 L_{\odot}$. This should be feasible with the deeper mid-IR surveys from Spitzer. For the more nearby $\rho$ Ophiuchi region $(d=140 \mathrm{pc})$, Bontemps et al. (2001) found the IMF of Class II sources (complete to $0.03 L_{\odot}$ ) to be well fitted by a two-step power-law with indices $\Gamma=-1.7$ for $m>0.55 M_{\odot}$ and $\Gamma=-0.15$ for the whole interval $0.055 M_{\odot}<m<0.55 M_{\odot}$ (accounting for unresolved binaries gives $\Gamma=-0.35)$. Thus, there are indications that the Class II IMFs are better described by the Scalo (1998) or the Kroupa et al. (1993) than by the more recent Kroupa (2001). 


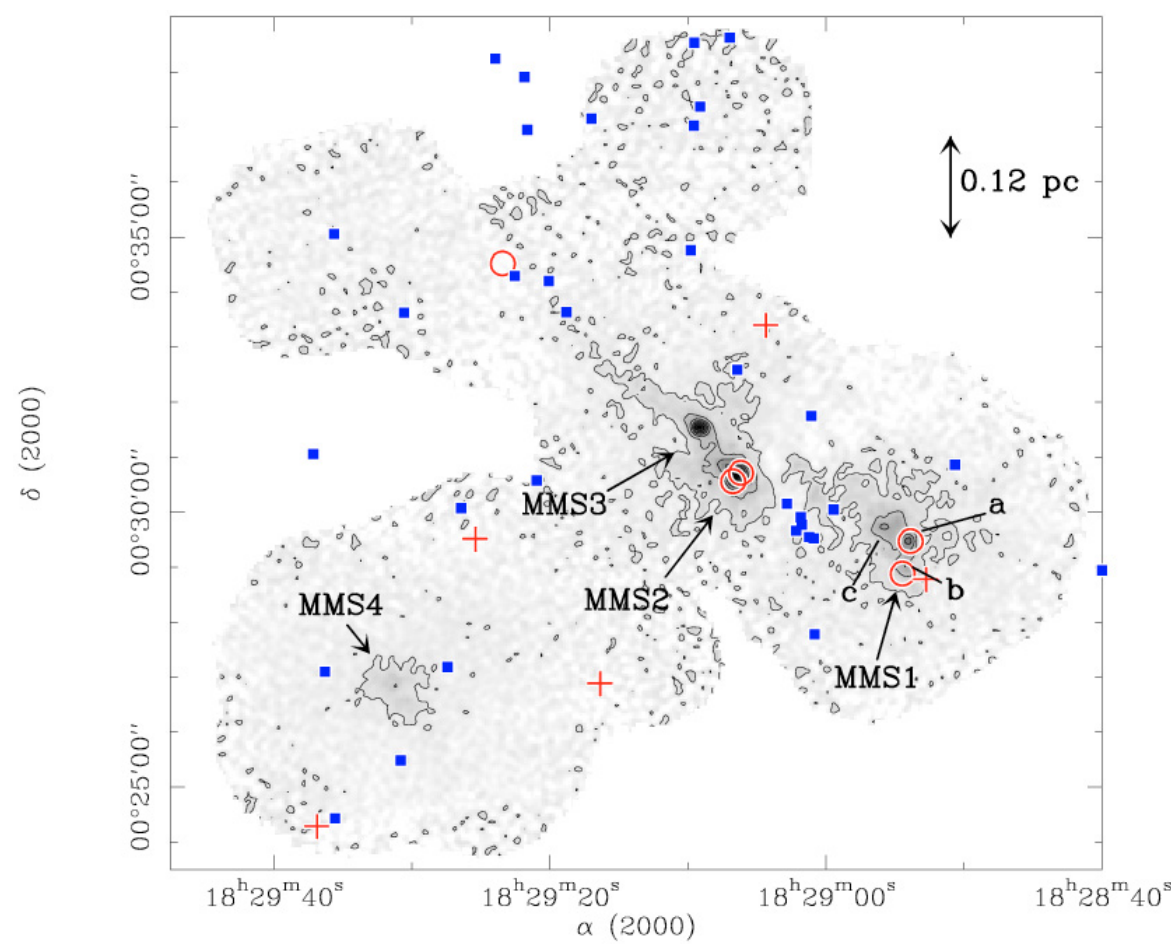

Fig. 12. The IRAM $1.3 \mathrm{~mm}$ continuum map with the location of the YSOs classified in this paper marked. The contour levels go from 30 to $450 \mathrm{mJy} / 11^{\prime \prime}$-beam in steps of $60 \mathrm{mJy} / 11^{\prime \prime}$-beam. The rms noise is $9 \mathrm{mJy} / 11^{\prime \prime}$-beam in the best parts of the image and typically around $15 \mathrm{mJy} / 11^{\prime \prime}$ beam on average. Class I sources (red circles), flat-spectrum sources (red crosses), and Class II sources (blue filled squares) are indicated. The four bright mm sources are labelled MMS1, MMS2, MMS3, and MMS4. The 0.12 pc clustering scale for protostars found in Kaas et al. (2004) is shown.

\section{Cloud structure deduced from $1.3 \mathrm{~mm}$ and $3.6 \mathrm{~cm}$ observations}

Figure 12 shows the IRAM $1.3 \mathrm{~mm}$ continuum mapping of a major part of the ISOCAM field. Four strong continuum sources are labelled in the figure. One is a multiple of three and another is elongated - all listed with coordinates and fluxes in Table 7 . The protostar clustering scale of $0.12 \mathrm{pc}$ found in the Serpens Cloud Core (Kaas et al. 2004) is drawn in the map for size reference. Also shown are the positions of YSO candidates found in Sect. 4: Class I sources, flat-spectrum sources, and Class II sources.

The contour plot of the $1.3 \mathrm{~mm}$ continuum emission outlines the position of the two ammonia cores found by Clark (1991) on each side of the optically visible stellar group Ser/G3-G6 quite well. MMS4 is a starless core coinciding with an absorption core seen in both ISOCAM filters. It is a candidate prestellar core similar to the cores studied in absorption with ISOCAM by Bacmann et al. (2000). MMS1 is located in the Ser/G3-G6SW ammonia core and is a multiple source whose brightest components we have denoted as a, b, and c. Both MMS2, which is extended, and MMS3 are located in the Ser/G3-G6NE ammonia core.

Morphologically, the $1.3 \mathrm{~mm}$ map is quite similar to the IRAM map of the Serpens Cloud Core (Kaas et al. 2004), showing two separate clumps of which one has an elongated tail in the direction of the alignment of the clumps along an elongated density structure or a cloud filament. Here the elongated density structure oriented NE-SW is also well traced in the $K$-band image of Fig. 2 as a pronounced drop in the surface density of faint stars. In the case of the Serpens Cloud Core, the NW-SE oriented ridge was described in, e.g., Kaas (1999a). Each clump contains a sub-structure. This region differs from the Cloud Core, however, in that the number of protostars is lower, and the
Table 7. The bright IRAM $1.3 \mathrm{~mm}$ sources with $\mathrm{J} 2000$ positions good to about \pm 3 arcsec.

\begin{tabular}{lcrrr}
\hline \hline Name & $\alpha_{2000}$ & $\delta_{2000}$ & $\begin{array}{r}\text { Peak } S_{1.3 \mathrm{~mm}} \\
(\mathrm{mJy} / \mathrm{beam})\end{array}$ & $\begin{array}{r}\mathrm{Int}^{1} S_{1.3 \mathrm{~mm}} \\
(\mathrm{mJy})\end{array}$ \\
\hline MMS1-a & 182854.1 & 002929 & 234. & 830 \\
MMS1-b & 182854.3 & 002902 & 153. & 700 \\
MMS1-c $^{2}$ & 182855.8 & 002945 & 161. & 780 \\
MMS2 $^{2}$ & 182906.5 & 003041 & 404. & 1650 \\
MMS3 $^{1829} 09.2$ & 003132 & 399. & 1020 \\
MMS4 & 182931.2 & 002650 & 94. & 420 \\
\hline
\end{tabular}

1 Integrated over an aperture with 37 " diameter. ${ }^{2}$ This source is elongated.

$1.3 \mathrm{~mm}$ emission is in general less intense. The immediate interpretation is that we see no such intense burst of star formation here as in the Cloud Core, which is also in agreement with the Class I/Class II number fraction discussed at the end of Sect. 4.1.

\subsection{Individual $1.3 \mathrm{~mm}$ and $3.6 \mathrm{~cm}$ sources}

The fluxes and positions of the IRAM $1.3 \mathrm{~mm}$ and VLA $3.6 \mathrm{~cm}$ sources are given in Tables 7 and 8. Taking into account the uncertainties in the ISOCAM positions (estimated in Sect. 2.2) and the IRAM positions ( $\sim 3^{\prime \prime}$ on the average and $5^{\prime \prime}$ at most), here we compare the $\mathrm{mm}$ and $\mathrm{cm}$ source positions with those of ISOCAM.

ISO-62 is probably the IR counterpart of MMS1-a, as the coordinates agree to $1.8^{\prime \prime}$. There is no $K$-band detection, but $L^{\prime}=11.4 \mathrm{mag}$, which gives a mid-IR SED index $\alpha_{\mathrm{IR}}^{4-14}=$ 1.54 - consistent with a Class I source classification. The radio source VLA-\#2 is positioned only $3.8^{\prime \prime}$ away from the 
Table 8. The VLA $3.6 \mathrm{~cm}$ sources with $\mathrm{J} 2000$ positions good to \pm 0.5 arcsec. All except one are detections above $5 \sigma$. The beam size is given in Sect. 2.7.

\begin{tabular}{lccrr}
\hline \hline $\begin{array}{l}\text { VLA } \\
(\#)\end{array}$ & $\alpha_{2000}$ & $\delta_{2000}$ & $\begin{array}{r}S_{3.6 \mathrm{~cm}} \\
(\mathrm{mJy} / \text { beam })\end{array}$ & Associations \\
\hline 1 & 182853.49 & 002911.9 & 0.08 & \\
2 & 182854.05 & 002932.7 & 0.08 & MMS1-a, ISO-62 \\
$(3)^{1}$ & 182855.80 & 002943.6 & 0.05 & MMS1-c \\
4 & 182901.80 & 002954.4 & 3.30 & ISO-82, Ser-G3 \\
5 & 182903.31 & 003057.9 & 0.09 & \\
6 & 182906.70 & 003033.6 & 0.32 & ISO-94, MMS2 \\
7 & 182909.03 & 003130.6 & 0.08 & MMS3 \\
8 & 182911.14 & 002924.8 & 0.12 & ISO-107 \\
9 & 182925.52 & 002504.0 & 0.14 & \\
\hline
\end{tabular}

${ }^{1}$ This is a $3.3 \sigma$ detection only, and therefore only tentative.

$\mathrm{mm}$ source. The MMS1-b position differs by $8.5^{\prime \prime}$ from the IR coordinates of ISO-64, which is also a Class I source. Since this is just below the $3 \sigma$ in positional uncertainty, it can not be excluded that ISO-64 is the infrared counterpart of MMS1-b. At the position of MMS1-c we find no IR counterpart, although we note that this region suffered strongly from persistency effects in both ISOCAM maps. There is, however, a $3.3 \sigma$ radio source (VLA-\#3) only $1.4^{\prime \prime}$ to the south of MMS1-c. We tentatively suggest MMS1-c as a possible Class 0 candidate.

MMS2 is elongated, and its position determined by the peak emission is found between the ISOCAM positions of the two Class I sources ISO-90 and ISO-94. The MMS2 elongation is roughly in the direction towards the mid-IR sources, and we interpret these to be the IR counterparts of the elongated MMS2 source, possibly an unresolved double. (The offsets between the peak MMS2 coordinate and the ISOCAM positions are $7.8^{\prime \prime}$ and $12^{\prime \prime}$ from ISO-90 and ISO-94, respectively.) We also note that a radio source (VLA-\#6) is found at the position of ISO-94 offset by only 6.2". We note that the Arnica/NOT position of the $K_{\mathrm{S}}=15.5 \mathrm{mag}$ source (NOT-343) agrees within $0.9^{\prime \prime}$ with the VLA position.

MMS3 is separated from the faint mid-IR source ISO-101 by $6^{\prime \prime}$. ISO-101 has no $K$-band detection, and in the $L^{\prime}$-band only a very faint extended signal was seen. Judging from Fig. 16 the position of ISO-101 seems to coincide with the extended $\mathrm{H}_{2}$ line emission. Also, the ISOCAM colour [14.3/6.7] is rather blue compared to the lower limit on the $\alpha_{\mathrm{IR}}^{2-14}$ index. This is likely explained by $\mathrm{H}_{2}$ line emission in the $6.7 \mu \mathrm{m}$ band and suggests that ISO-101 is a just a hot spot in the bright jet-like feature seen in the deep $\mathrm{H}_{2}$ line image in Fig. 13, rather than being a continuum source. Most probably, ISO-101 is not the mid-IR counterpart of MMS3. There is a radio source (VLA-\#7) less than $3^{\prime \prime}$ from the IRAM position of MMS3. Based on this we propose that MMS3 is a new Class 0 candidate and probably the driving source of the bright outflow seen in the deep $\mathrm{H}_{2}$ line image in Fig. 13 (see discussion in Sect. 6.2).

MMS4 has no IR sources in its vicinity, and as described above it is a candidate prestellar core. The VLA source \#4 is a very bright radio source coinciding in position with ISO-82 (CoKu Ser-G3), a Class II source that is seen optically and was classified as a Classical T Tauri star by Cohen \& Kuhi (1979). The position of another radio source (VLA-\#8) is only $4.2^{\prime \prime}$ away from ISO-107, a bright ( $K=7.8 \mathrm{mag})$ star in the central part of the cluster that has no infrared excess, but could possibly be a YSO of type Class III. Three VLA sources (\#1, 5, and 9) are

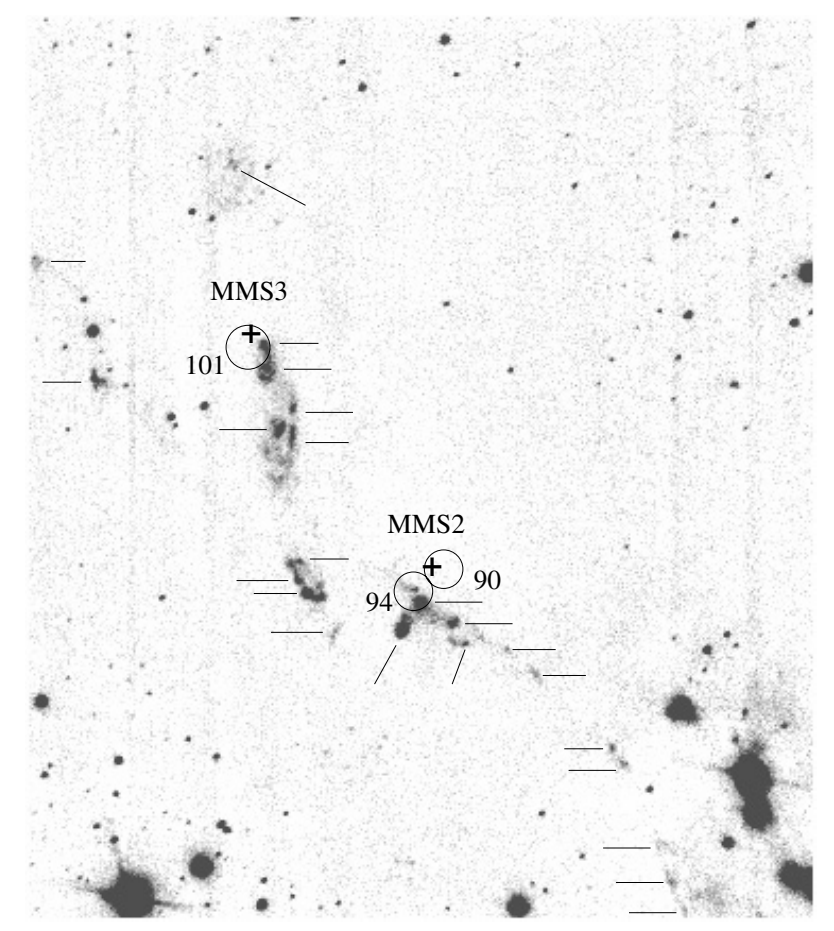

Fig. 13. NOTCam $\mathrm{H}_{2}$ line image $(2.122 \mu \mathrm{m})$ of Ser/G3-G6NE. Pure line emission features are arrowed (cf. the continuum image in Fig. 14). The locations of the two bright mm sources MMS2 and MMS3 are marked with plus signs. The Class I candidates ISO-90 and 94, as well as ISO-101, probably a hot spot in the jetlike emission, are encircled.

without $\mathrm{mm}$ or IR counterparts and are probably extragalactic sources.

Radio continuum emission at $3.6 \mathrm{~cm}$ has been detected for a number of YSOs - most of them in the Class 0 or Class I protostellar phase (Eiroa et al. 2005). Spectral indices obtained at $\mathrm{cm}$ wavelengths show that thermal free-free emission from ionised gas usually dominates, and it has been suggested that the radio emission from protostars is due to thermal radio jets ionized by shocks in strong stellar winds (Anglada et al. 1998; Reipurth et al. 2004). Although we have no information on the spectral index in the radio, this is probably the most likely interpretation for our VLA sources \#2, 3, 6, and 7, which all correspond to Class I or Class 0 sources, and of which two are associated with $\mathrm{H}_{2}$ outflows (see next section). For comparison, in the Serpens Cloud Core Eiroa et al. (2005) found that as many as 10 of 16 YSOs with radio emission were in the Class 0 to Class I phase, while only about $25 \%$ were as evolved as Class II sources. For this last group it has been suggested that non-thermal gyrosynchrotron emission from coronally active stars may explain the radio emission (Smith et al. 1999). We speculate that this could be the case for VLA \# 4 and 8, although we have no X-ray information for these sources. Determination of the radio spectral indices is needed to confirm the above suggestions.

\subsection{Outflows traced by the $2.122 \mu \mathrm{m} S(1)$ line of $\mathrm{H}_{2}$}

The Ser/G3-G6NE ammonia core was imaged with NOTCam in the $2.122 \mu \mathrm{m} \mathrm{S}(1)$ line of $\mathrm{H}_{2}$ (see Fig. 13). Numerous Herbig-Haro $(\mathrm{HH})$ objects with pure $\mathrm{H}_{2}$ line emission are found by comparison with the $2.087 \mu \mathrm{m}$ continuum image in Fig. 14. The morphology is complex, and proper motion of the knots is not yet available, but the features probably arise from at least two different bipolar collimated flows crossing each other along 


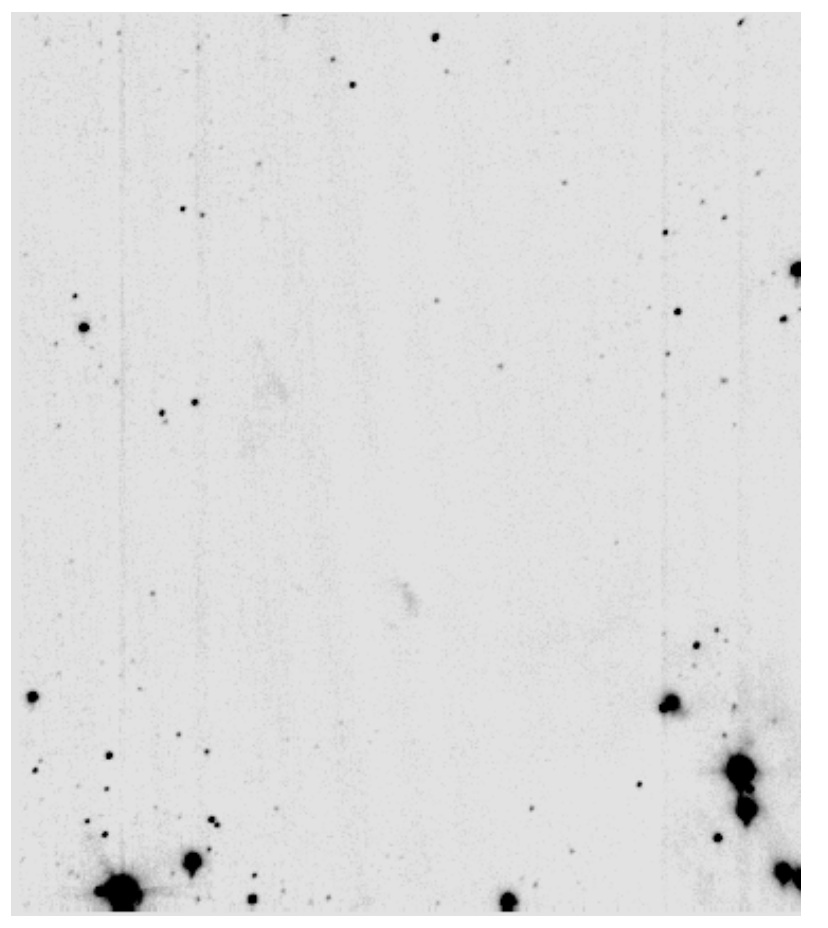

Fig. 14. NOTCam $2.087 \mu \mathrm{m}$ continuum image of Ser/G3-G6NE. The same field as Fig. 13.

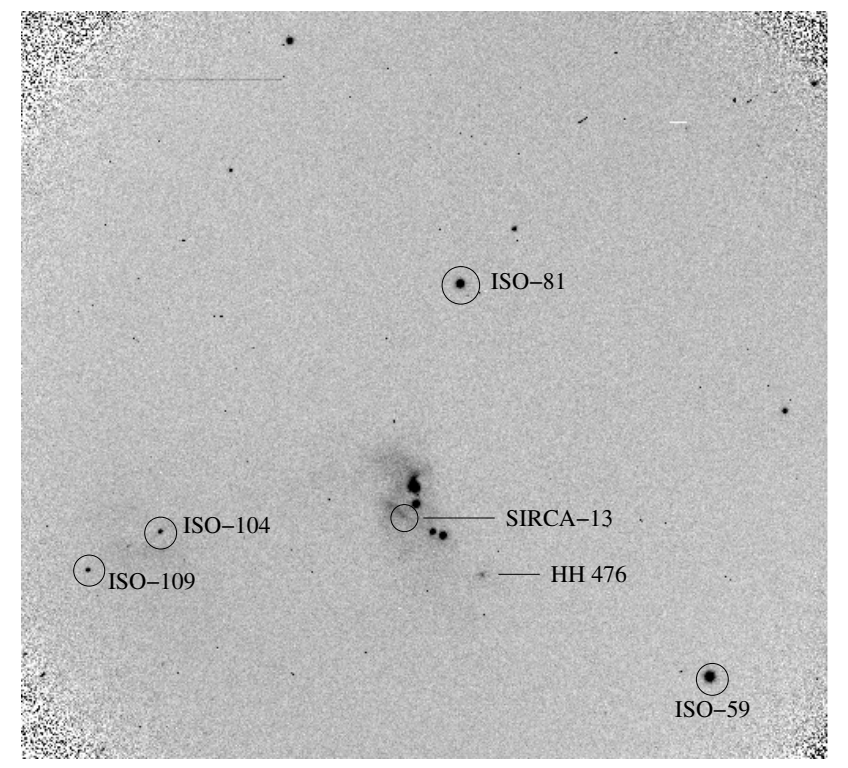

Fig. 15. ALFOSC/NOT image of 900 s integration through the $\mathrm{H}_{\alpha}$ filter (18 nm wide) in June 2002. Apart from some extended emission around the Ser/G3-G6 group, the only pure line emission object seen in the optical is the already known HH 476. FOV $=6.5^{\prime}$.

the line of sight. From the optical $\mathrm{H}_{\alpha}$ image in Fig. 15, it is evident that the flows are deeply embedded. We tentatively suggest that the driving sources are the two dense mm cores MMS2 and MMS3, the last one proposed to be a new Class 0 candidate.

Among the two bipolar flows traced by the numerous $\mathrm{HH}$ objects seen in the near-IR in Fig. 13, one is a very long collimated flow oriented NE-SW and extending over the full FOV of the image. It probably arises from MMS2, either of the possible multiple or as a consequence of disintegrating multiples, a mechanism suggested by Reipurth (2000) and Reipurth et al. (2004). Its extension is at least $90^{\prime \prime}$ to both sides of the driving source. We

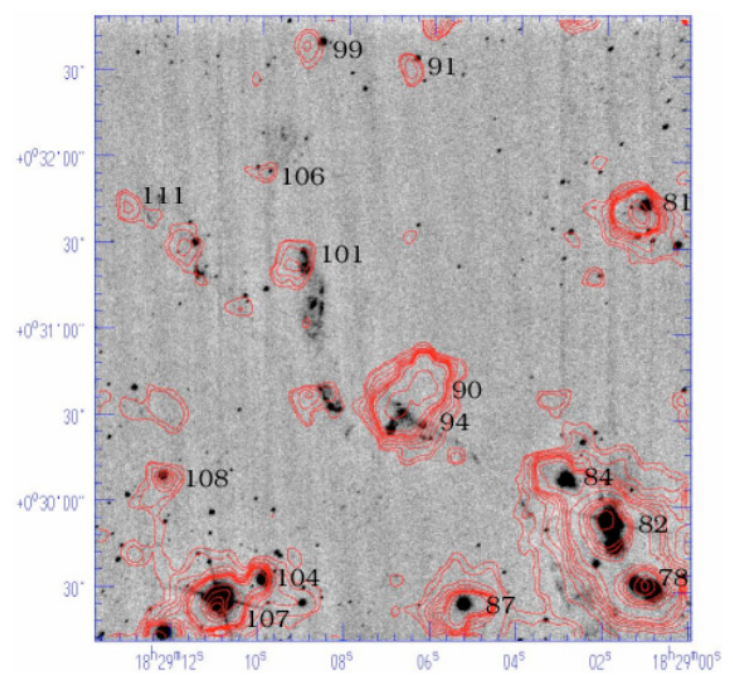

Fig. 16. NOTCam $\mathrm{H}_{2}$ line image $(2.122 \mu \mathrm{m})$ of the Ser/G3-G6NE core. ISOCAM $6.7 \mu \mathrm{m}$ contours are overlaid, and ISOCAM sources are labelled with the ISO ID number of Table 1.

notice that the flow is slightly S-shaped. In the NE it ends in a bow-shock-like morphology (upper left of image). The optical Herbig-Haro object $\mathrm{HH} 476$ that falls just outside the $\mathrm{H}_{2}$ image (but is shown in the larger field $\mathrm{H}_{\alpha}$ image in Fig. 15) may be related to this flow. HH 476 was detected by Ziener \& Eislöffel (1999) in an optical [SII] survey, and Wu et al. (2002) suggested its energy source to be IRAS $18265+0028$, which ISOCAM resolved in the two very red sources ISO-90 and ISO-94. Followup imaging is needed to determine the proper motion of the knots and to confirm our tentative interpretation.

The remaining $\mathrm{H}_{2}$ emission features, i.e., the bright knots to the south of MMS3 and grossly aligned almost N-S, and also the faint extended emission seen to the north of MMS3 around the position of ISO-106, most likely arise from another outflow, one that is probably driven by MMS3, a new Class 0 candidate. The morphology is more complex with ringlike shapes, and more data is needed to interpret these structures.

The only extended continuum radiation seen in Fig. 14 is some faint nebulous emission in the vicinity of the two suggested driving sources, MMS2 and MMS3, and this is probably scattered light.

\section{Summary and conclusions}

An embedded cluster of YSOs was found from an ISOCAM survey around the optical stellar group Ser/G3-G6. Mid-IR photometry in two broadband filters centred on 6.7 and $14.3 \mu \mathrm{m}$ obtained with ISOCAM was combined with data from various telescope/instrument configurations to present a multiwavelength study. This reveals low-mass YSOs in the evolutionary stages from Class 0 to Class III. We propose 2 Class 0 candidates, 5 Class I candidates, 5 flat-spectrum sources, 31 Class II objects, and 3 Class IIIs (with some IR-excess). Our selection criterium is IR excess, which means that our sample is severly incomplete for Class III sources in general. This also means that we cannot estimate the disk fraction in the YSO population. The number fraction of Class I to Class II sources in the Ser/G3-G6 IR cluster (5/31) is quite typical for star-forming regions and comparable to the $\rho$ Ophiuchi and Chamaeleon I clusters, i.e., quite different from the unusually large fraction of Class Is found in the Serpens Cloud Core (Kaas et al. 2004). 
The IRAM $1.3 \mathrm{~mm}$ continuum mapping detects several bright continuum sources in the two ammonia cores Ser/G3-G6NE and Ser/G3-G6SW, and one outside that is suggested as a candidate prestellar core. MMS1-c and MMS3 are proposed to be Class 0 candidates. Both of them are also VLA $3.6 \mathrm{~cm}$ radio continuum sources. Deep NOTCam imaging in the $2.122 \mu \mathrm{m}$ line of $\mathrm{H}_{2}$ reveals signs of two complex outflows in the Ser/G3-G6NE core. A deep $\mathrm{H}_{\alpha}$ image shows that the flows are embedded and only the previously known HH 476 is seen optically. We suggest that the driving sources of these two outflows are MMS2 and MMS3.

Comparing with existing pre-main sequence evolutionary models, we find that for any reasonable assumptions on age for the Class II sources, our sample extends well into the BD mass regime. The Class II sample was joined with the Class II sample from the Serpens Cloud Core to improve statistics. Model LFs calculated for a set of ages and IMFs give a best age of $2 \mathrm{Myr}$ for which both the Scalo (1998) and Kroupa (2001) IMFs are compatible with the observed LF down to the completeness limit at $0.08 L_{\odot}$. The Class II LF can be described by co-eval star formation 2 Myr ago, continuous star formation over the last $2 \mathrm{Myr}$, or a burst of star formation of various duration that took place $2 \mathrm{Myr}$ ago. There is a peak in the observed LF that cannot be used to constrain the age because it coincides with the completeness limit. Nevertheless, the general shape of the LF does not permit a much younger or older population. The completeness limit must be shifted to $0.04 L_{\odot}$ to possibly distinguish between different IMFs and star formation scenarios. This should be feasible with the deeper mid-IR surveys now available from Spitzer.

Acknowledgements. We thank the referee, Dr. Paul Harvey, for suggestions that led to substantial improvements of the paper. We acknowledge Dr. F. O. Clark for presenting his unpublished $\mathrm{NH}_{3}$ mapping to us before planning the ISOCAM observations. The ISOCAM data presented in this paper was reduced using "CIA", a joint development by the ESA Astrophysics Division and the ISOCAM Consortium led by the ISOCAM PI, C. Cesarsky, Direction des Sciences de la Matiere, C.E.A., France. This work is partly based on observations made with the NOT, operated on the island of La Palma jointly by Denmark, Finland, Iceland, Norway, and Sweden, in the Spanish Observatorio del Roque de los Muchachos of the Instituto de Astrofisica de Canarias. One image presented here has been taken using ALFOSC, which is owned by the Instituto de Astrofisica de Andalucia (IAA) and operated at the NOT under an agreement between IAA and the NBIfAFG of the Astronomical Observatory of Copenhagen. This publication makes use of data products from the Two Micron All Sky Survey, which is a joint project of the University of Massachusetts and the Infrared Processing and Analysis Center/California Institute of Technology, funded by the National Aeronautics and Space Administration and the National Science Foundation. This research has made use of the SIMBAD database, operated at CDS, Strasbourg, France, as well as SAOImage DS9, developed by the Smithsonian Astrophysical Observatory.

\section{References}

Abbott, T. M. C., Aspin, C., Sørensen, A. N., et al. 2000, Proc. SPIE, 4008, 714 Adams, F. C., Lada, C. J., \& Shu, F. H. 1987, ApJ, 312, 788

Anglada, G., Villuendas, E., Estalella, R., et al. 1998, AJ, 116, 2953

André, P., \& Montmerle, T. 1994, ApJ, 420, 837

André, P., Ward-Thompson, D., \& Barsony, M. 1993, ApJ, 406, 122

André, P., Ward-Thompson, D., \& Barsony, M. 2000, in Protostars and Planets IV, ed. V. Mannings, A. P. Boss, \& S. S. Russell (Tucson: University of Arizona Press), 59

Bacmann, A., André, P., Puget, J.-L., et al. 2000, A\&A, 361, 555

Baraffe, I., Chabrier, G., Allard, F., \& Hauschildt, P. H. 1998, A\&A, 337, 403

Bessell, M. S., \& Brett, J. M. 1988, PASP, 100, 1134

Blommaert, J., Metcalfe, L., Altieri, B., et al. 2000, Experimental Astronomy, 10,241

Bonnell, I. A., Larson, R. B., \& Zinnecker, H. 2006, in Protostars and Planets V, ed. B. Reipurth, D. Jewitt, \& K. Keil, in press [arXiv:astro-ph/0603447]

Bontemps, S., André, P., Kaas, A. A., et al. 2001, A\&A, 372, 173

Broguière, D., Neri, R., \& Sievers, A. 1995, NIC bolometer users guide (IRAM internal report)
Casali, M. M., \& Hawarden, T. G. 1992, UKIRT Newsletter, 4, 33

Clark, F. O. 1990, private communication

Clark, F. O. 1991, ApJS, 75, 611

Cesarsky, C. J., Abergel, A., Agnèse, P., et al. 1996, A\&A, 315, L32

Cohen, M., \& Kuhi, L. V. 1979, ApJS, 41, 743

Curiel, S., Rodríguez, L. F., Moran, J. M., \& Cantó, J. 1993, ApJ, 415, 191

Cutri, R. M., Skrutskie, M. F., Van Dyk, S., et al. 2003, Explanatory Supplement to the 2MASS All Sky Data Release,

http://www.ipac.caltech.edu/2mass/releases/allsky/doc/ explsup.html

Dame, T. M., \& Thaddeus, P. 1985, ApJ, 297, 751

D’Antona, F., \& Mazzitelli, I. 1998, in Brown Dwarfs and Extrasolar Planets, ed. R. Rebolo, E. L. Martin, \& M. R. Zapatero Osorio, ASP Conf. Proc., 134, 442

Emerson, D. T., Klein, U., \& Haslam, C. G. T. 1979, A\&A, 76, 92

Eiroa, C., Torrelles, J. M., Curiel, S., \& Djupvik, A. A. 2005, AJ, 130, 643

Eiroa, C., Djupvik, A. A., \& Casali, M. M. 2006, in Handbook of star formation regions, ed. B. Reipurth, in press

Folha, D. F. M., \& Emerson, J. P. 1999, A\&A, 352, 517

Froebrich, D., Ray, T. P., Murphy, G. C., \& Scholz, A. 2004, A\&A, 432, L67

Greene, T. P., Wilking, B. A., André, P., Young, E. T., \& Lada, C. J. 1994, ApJ, 434,614

Griffin, M. J., \& Orton, G. S. 1993, Icarus, 105, 337

Harvey, P. M., Chapman, N., Lai, S.-P., et al. 2006, ApJ, in press

Hunt, L., Mannucci, F., Testi, L., et al. 1998, AJ, 115, 2594

Kaas, A. A. 1999 a, AJ, 118, 558

Kaas, A. A. 1999b, Ph.D. Thesis, Stockholm University, Akademitryck AB, Edsbruk

Kaas, A. A., \& Bontemps, S. 2000, in From Darkness to Light: Origin and Evolution of Young Stellar Clusters, ed. T. Montmerle, \& P. André, ASP Conf. Proc., 243, 367

Kaas, A. A., Olofsson, G., Bontemps, S., et al. 1999, in The Universe as seen by ISO, ed. P. Cox, \& M. F. Kessler, ESA SP-427, 493

Kaas, A. A., Olofsson, G., Bontemps, S., et al. 2004, A\&A, 421, 623

Kessler, M. F., Steinz, J. A., Anderegg, et al. 1996, A\&A, 315, L27

Koornneef, J. 1983, A\&A, 128, 84

Kreysa, E., Gemünd, H.-P., Gromke, et al. 1998, in Advanced Technology MMW, Radio, and Terahertz Telescopes, ed. T. G. Phillips, Proc. SPIE, 3357, 319

Kroupa, P. 2001, MNRAS, 322, 231

Kroupa, P., Tout, C., \& Gilmore, G. F. 1993, MNRAS, 262, 545

Lada, C. J. 1987, in Star Forming Regions, ed. M. Peimbert, \& J. Jugaku (Dordrecht: D. Reidel Publishing Co.), IAU Symp., 115, 1

Lada, C. J., \& Wilking, B. A. 1984, ApJ, 287, 610

Lawson, W. A., Crause, L. A., Mamajek, E. E., \& Feigelson, E. D. 2002, MNRAS, 329, L29

Lyo, A.-Ran, Lawson, W. A., Mamajek, et al. 2003, MNRAS, 338, 616

Meyer, M. R., Calvet, N., \& Hillenbrand, L. A. 1997, ApJ, 114, 288

Olofsson, G., Kaas, A. A., Nordh, L., et al. 1998, in Brown Dwarfs and Extrasolar Planets, ed. R. Rebolo, E. L. Martin, \& M. R. Zapatero Osorio, ASP Conf. Proc., 134, 81

Olofsson, G., Florén, H.-G., \& Lagage, P. O. 1999, Imaging in the Thermal Infrared, in Astrophysics with the NOT, ed. H. Karttunen, \& V. Piirola (University of Turku), 37

Persi, P., Palagi, F., \& Felli, M. 1994, A\&A, 291, 577

Persi, P., Marenzi, A. R., Olofsson, G., et al. 2000, A\&A, 357, 219

Reipurth, B. 2000, AJ, 120, 3177

Reipurth, B., Rodríguez, L. F., Anglada, G., \& Bally, J. 2004, AJ, 127, 1736

Salpeter, E. 1955, ApJ, 121, 161

Scalo, J. 1998, in The Stellar Initial Mass Function, ed. G. Gilmore, \& D. Howell, Astronomical Society of the Pacific, 142, 201

Smith, K., Güdel, M., \& Benz, A. O. 1999, A\&A, 349, 475

Straižys, V., Černis, K., \& Bartašiūtè, S. 2003, A\&A, 405, 585

Strom, K. M., Strom, S. E., \& Merrill, K. M. 1993, ApJ, 412, 233

van der Bliek, N., Olofsson, G., Florén, H.-G., et al. 2000, SIRCA, The Stockholm Infrared Camera, in The NOT in the 2000's, ed. N. Bergvall, L. Takalo, \& V. Piirola, University of Turku, 151

Whittet, D. C. B. 1988, The observed properties of interstellar dust in the infrared, in Dust in the Universe, ed. M. E. Bailey, \& D. A. Williams (New York: Cambridge University Press), 25

Wu, J. W., Wu, Y. F., Wang, J. Z., \& Cai, K. 2002, Chin. J. Astron. Astrophys., 2,33

Zhang, C. Y., Laureijs, R. J., Clark, F. O., \& Wesselius, P. R. 1988, A\&A, 199, 170

Ziener, R., \& Eislöffel, J. 1999, A\&A, 347, 565

Zinnecker, H., McCaughrean, M. J., \& Wilking, B. A. 1993, in Protostars and Planets III, ed. E. Levy, \& J. I. Lunine (Tucson: University of Arizona Press), 429 